\title{
Über die Endergebnisse der direkten Verletzungen der großen Gelenke.
}

\author{
Yon \\ Privatdozent Dr. Philipp Erlacher, \\ Leiter der chirurg.-orthopäd. Abtlg. der Einderklinik, Graz. \\ (Aus lem Wiener orthopädischen Spital. [Direktor Professor H. Spitzy.])
}

(Mit 7 'labellen und 17 Abbildungen im Text.)

(Eingegangen am 8. Februar 1920.)

Die direkten Verletzungen der Gelenke, die schon im Frieden einer besonderen Behandlung und Pflege bedurften, sind natürlich auch im Kriege ein Gegenstand besonderer Aufmerksamkeit in der Behandlung geworden, namentlich da ja die meisten dieser Kriegsgelenkverletzungen mehr oder minder schwer infiziert waren. Die besondere Empfindlichkeit der Gelenke gegen die Infektion und die meist schweren, das Leben bedrohenden Folgeerscheinungen, die trotz der größten Mühe zum Verlust des Gliedes führen können, haben zu eingehendem Studium angeeifert und auch eine Reihe von Behandlungsmethoden gezeitigt, die in erster Linie das Erhalten des Lebens, in zweiter linie das der ganzen Extremität und erst in dritter Linie eine möglichst große Beweglichkeit des Gelenkes zum Ziele haben. Da die einzelnen Statistiken meist nur auf Grund der Beobachtungen mit einer bestimmten Behandlungsmethode, fast immer unter ganz bestimmten gleichbleibenden żußeren Umständen aufgebaut sind und miteinander kaum übereinstimmen, dürfte es angebracht sein, an einem großen Material, wie es sich uns in einer großen Nachbehandlungsanstalt mit bereits 24000 Patienten bietet, zu untersuchen, welche Methoden im allgemeinen geübt werden, welche Erfolge sie zu erbringen vermögen und schließlich wie die Erfolge nach den einzelnen Gelenken bei den direhten Gelenksverletzungen an unseren Kriegsbeschädigten überhaupt sind. Ich habe daher unser gesamtes Waterial daraufhin untersucht und alle röntgenologisch oder durch die Inspektion festgestellten Verletzunger der großen Gelenke Schulter-, Ellbogen- und Handgelenk, Hüft-, Knie- and Fuß- (oberes und unteres Sprung-) gelenk untersucht und so eine Úbersicht über 1539 Fälle erhalten. Dabei ist jedoch zu bedenken, daß Fälle, die trotz einer Gelenkrerletzung mit vollständiger Funktionsfähigkeit ausgeheilt sind, also die besten und günstigsten Fäle, nicht 
aufgenommen sind, weil solche Fälle naturgemäß unserem Spitale überhaupt nicht erst zur Nachbehandlung zugcwiesen werden; anderseits wiederum kann ich auch über die Mortalität der Gelenkschüsse keinerlei Aufschlïsse geben, weil so schwer geschädigte Fälle den Weg bis zu uns nicht mehr gefunden haben.

\section{Gelenkverletzungen mit Opfermog der Extremität.}

Wohl aber habe ich versucht über das Verhältnis der primären Gelenkverletzung (wieder nur der genannten Gelenke) und der Amputationen einen Einblick zu bekommen, indem ich 1000 Amputierte anamnestisch auf eine Gelenkverletzung genauestens ausforschen ließ und dabei die relativ geringe Zahl von 120 Fällen feststellen konnte. Also $12 \%$ aller Amputationen müsseu im Anschluß oder infolge einer direkten Geleukverletzung ausgeführt werden. Von diesen 120 Fällen wurden 18, also $15 \%$ noch innerhalb der ersten 24 Stunden amputiert. Dies dürften wohl jene schwere Zertrümmerungen sein, bei denen das Gelenk als solches ganz zerstört war; die Amputation ist in diesen Fällen weniger als die Folge der Gelenks verletzung aufzufassen als vielmehr als eine Folge der schweren Zertrümmerung der Extremität überhaupt und nur zufällig an der Stelle eires Gelenkes. Somit bleiben als Amputationen infolge der Gelenkverletzungen nur ungefähr $10 \%$ aller. W etere 30 Amputationen waren in der ersten Woche erfolgt, hingegen 72 Fälle erst spaiter amputiert worden. bis zu 6 Wochen nach der Verletzung. Bei einem Drittel aller Fälle $(33,3 \%)$ waren vor der Amputation mehr oder minder große Eingriffe, wie Inzisionen. Drainage, Ausräumung, Splitterentfernungen und Aufklappungen (3 mal am $\mathrm{Knie}$ ) und eine Resektion (Knie) vorgenommen worden. Eine t'bursicht über die Beteiligung der einzelnen Gelenke gibt nachstehende Tabelle I.

Tabelle I.

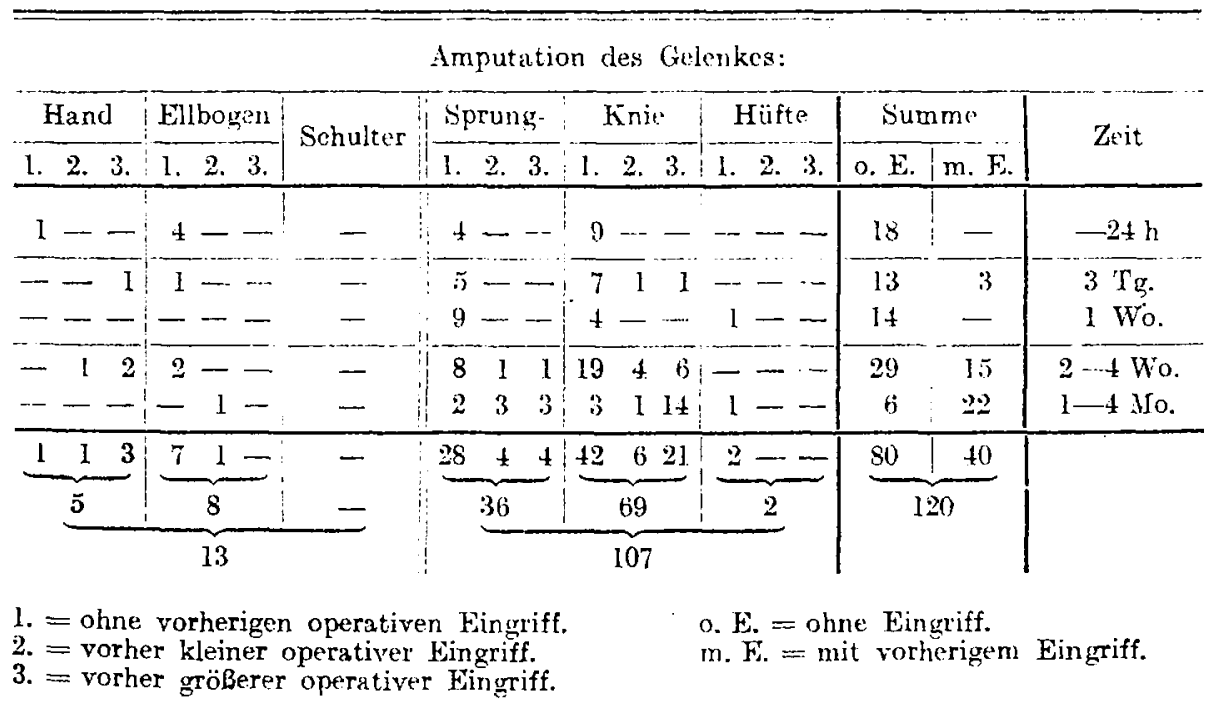


Wenn also auch diese Statistik keine Ubersicht darüber gibt, auf wie viel direkte Gelenkverletzungen eine Amputation kommt, so erfahren wir doch aus derselben, daß die Beteiligung ron Schulter und Hüfte (als Amputationsgrund) kaum in Betracht kommt, wohl deshalb, weil schwere Verletzungen dieser Gelenke auch mit schweren Zerreißungen der dort verlaufenden großen Gafäße verbunden sind und daher den Tod durch Verbluten in unmittelbaren Gefolge haben und nur weniger schwere Falle in ärztliche Behandlmg urd schließlich als Amputierte zu uns gelangen dürften. Dagegen sind die Amputationen in mehr als der Hälfte $(\tilde{5} 7,5 \%)$ wegen Knieverletzungen notwendig geworden und in 36 Fällen, also $30 \%$ wegen Verletzungen des Fußgelenkes. I m a 11 gemeinen kann man daraus schließen, daß die Verletzungen der großer Gelenke dor unteren Extremitat im Verhältnis von 9:1 zu den großen Golenken der oberen Extremität eine spätere Amputation erfordern und daB ferner die großen Gelenke nathe am Stamm: Schulter und $\mathrm{H}$ ïfte, kaum zu einer Amputation An la beben bzw. dadurch gerettet werden können.

Wenn wir meine Statistiken, die über die Amputationen nach Gelenkverletzungen und die nachstehende der geheilten Fälle miteinander vergleichen, so ergibt dies eine eigenartige Ergänzung. indem wir hier in der Zahl der Fälle der oberen und unteren Extremität ein umgekehrtes Verhältnis haben, nämlich 6: 4 der geheilten gegen 1:9 der Amputierten oder mit anderen Worten: von den Gelenkverletzungen der unteren Extremität muß eine größere Anzahl amputiert werden, an der oberen nur wenige; daher sind auch die geheilten Fälle bei der oberen Extremität entsprechend häufiger als an der unteren. Ein genaueres Bild der Beteiligung der einzelnen Gelenke gibt die nachstehende Tabelle II.

Tabelle Ir.

\begin{tabular}{|c|c|c|c|c|c|c|c|}
\hline \multicolumn{8}{|c|}{ Befund nach Verletzung des Gelenkes vou } \\
\hline Hand & Ellbogen & Schulter & FußB & Kusie & Hïfte & Sunme & \\
\hline 72 & 234 & 86 & $1+1$ & $1 ; 39$ & 6.5 & 737 & Ankylosen \\
\hline 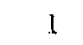 & $4: 3$ & 46 & \pm & 8 & It & 116 & Schlottergelenko \\
\hline 19 & 37 & 40 & 27 & 74 & 12 & 209 & stark / einge- \\
\hline 27 & 212 & 107 & 56 & 36 & 17 & 455 & mäßig $\begin{array}{c}\text { Beweglifch } \\
\text { keit }\end{array}$ \\
\hline 4 & 4 & 5 & 4 & 3 & 2 & 22 & normal \\
\hline 123 & 530 & 284 & 232 & 260 & 110 & 1539 & Summo \\
\hline $33^{\circ}$ & $57^{0}$ & $511^{\circ}$ & $26^{\circ}$ & $55^{0}$ & $\left(90^{\circ}\right)$ & & $\begin{array}{l}\text { Durchschnitt. } \\
\text { liches Bewe- } \\
\text { gungs-Ausma } 3 .\end{array}$ \\
\hline $\mathscr{H}^{0}$ & $96^{2}-126^{\circ}$ & $40^{\circ}$ & $112-140^{\circ}$ & $180^{\circ}$ & $\begin{array}{l}\text { gestreckt } \\
10^{\circ} \text { Ad- } \\
\text { duktion }\end{array}$ & & $\begin{array}{l}\text { Häufigster Ver- } \\
\text { steifungswinkel. }\end{array}$ \\
\hline
\end{tabular}




\section{Gelenkverletzungen bei Rettung der Extremität.}

Die 1539 Fälle teilen sich also in 937 Celenkverletzungen der oberen und 602 der unteren Extremität. Weitaus die größte Zahl, über ein Drittel aller Fälle, stellt der Ellbogen bei $(34,46 \%)$, dam kommen ungefähr gleich stark die Schulter mit 18,466\%, das Knie mit 16,84\% und das FuBgelenk mit 15,084\%; sehr gering ist die Beteiligung der Hand mit fast $8 \%$ und der Hüfte mit $7,152 \%$. Als Endergebnis der Behandlung findet sich in fast der Hälfte aller Verletzungen $(47,92 \%)$ eine feste knöcherne Ankylose, wobei die Gelenke der unteren Extremität mit durchschnittlich $58 \%$ gegenüber nur $44 \%$ der oberen, wesentlich stärker beteiligt sind, ist wohl darauf zurückzufïhren, daß die Gelenkenden beim Gebrauch des Beines aneinander gepreßt werden; mit mehr oder minder schweren Kontrakturen sind 43,109\% ausgeheilt. Relativ groß ist die Zahl der Schlottergelenke 7,542\%, die fast durchwegs sehr schweren Grades sind, hingegen beträgt die Zahl der normalen Fälle entsprechend dem Charakter unserer Anstalt nur 22 Fälle oder $1,43 \%$. Das Ausmaß an Beweglichkeit, das beiden einzelnen direkt verletzten Gelenken erzielt werden konn te, beträgt, a bgesehen von den vereinzelten Fällen mit normalem Bewegungsumfang beim Ellbogengelenk $57^{\circ}$, beim Kunie $55^{\circ}$, für die Schulter $51^{\circ}$, dagegen nur $33^{\circ}$ für die Hand und $26^{\circ}$ für das Fußgelenk, jedoch fast $90^{\circ}$ für das Hüftgelenk. Die Zahl für die Hüfte ist jedoch für eine allgemein gültige Beurteilung des Erfolges bei Hüftverletzungen nicht zu verwerten, weil sie sich nur a uf 5 Fälle bezieht und weil die Messungen in Hüftgelenk außerordentlich schwierig sind. Im allgemeinen kann man aber jedenfalls feststellen, daß die Ankylosierung des Hand-, Ellbogen- und Kriegelenkes in funktionell brauchbarer Stellung erfolgt ist, und man muß das Bewegungsausma 3 jencr Fälle, bei denen trotz der direkten Verletzung des Gelenkes eine Beweglichkeit erzielt werden konnte, als für die Funktion des Gliedes ausreichend bezeichnen, nur für die Schulter muß ein Heben um nur $50^{\circ}$ - trotz Unterstïtzung durch die Schultergürtelmuskein! - als sehr gering festgestellt worden, während für das Fußgelenk der oft extreme Spitzfuß cin schweres Hindern is für eine gute Gebrauchsfähigkeit darstellt. Besonders hervonzuheben ist auBerdem noch die Zahl der Schlottergelenke bei Schulter und Ellbogen, und zwar deshalb, weil sie gerade in diesen beiden Gelenken die Gebralichsfähigkeit der Extremitat so außerordentlich schädigen, die deshalb leichter entstehen, weil die Schwere die Gelenkenden auseinander zieht, während dagegen die Schlottergelenke der Hüfte in 14 Fïllen funktionell kaum störerd in Erscheinung traten. t'ber die chirurgische Bchandlungsweise der geheilten Falle: wäre nur noch zu erwähnen, daß sie nur zum geringen Teil Spuren zielbewußter operativer Eingriffe zeigten, die im Anschluß an die primäre Verletzung vorgenommen worden waren; darunter sind Resektionen in geringer Zahl wohl an allen Gelenken und einige Aufklappungen am Kniegelenk.

Besonderer Teil.

Handgelenk. (Kurve la, b.)

Die direkten Verletzungen des Handgelenkes sind nicht allzuhäutig und nehmen mit 123 Füllen nur $8 \%$ meiner Gelenkverletzungen ein. Ich möchte dabei betonen, daß ich nur die schwereren "Fälle aufgenommen habe, währerd ich Fälle mit lediglich einem Abbruch z. B. des proc. styloid. radii oder ulnae, 
die ja streng genommen auch das Handgelenk betreffen, ausgeschieden habe. Eine typische Behandlungsart konnte ich nicht feststellen. In den meisten Fällen hatte wohl die Schußverletzung als solche für genügend freien Sekretabfluß gesorgt. War die Eiterung fortgeschritten, so fanden sich Inzisionen am Unterarm; in 4 Fällen war eine Resektion des Gelenkes vorgenommen worden, und in weiteren 3 Fällen war das Gelenk durch die Verletzung selbst auf das schwerste zertrümmert, die Knochenteile zum Teil entfernt worden. Diese 7 Fälle von grober Zerstörung des Gelenkes waren mit einem Grenze der Hebung Schlottergelenk und 6 festen Ankylosen ausgeheilt. Im ganzen fand ich 72 Ankylosen, also $58,53 \%$, I Schlottergelenk, in 19 Fällen war die Beweglichkeit sehr gering, in 27 war sie noch wesentlich herabgesetzt und nur in 4 Fällen normal. Im allgemeinen scheinen alle Handgelenkverletzungen auf eine gerade Schiene gelagert zu werden, denn ich fand unter den 72 Ankylosen nur 5 in Beugestellung versteift, davon allerdings 2 unter einem Winkel von $110^{\circ}$ und je eine unter $140^{\circ}, 160^{\circ}$ und $168^{\circ}$, alle anderen in gerader Streckstellung. Hingegen erscheint öfter je nach der Mitbeteiligung der Elle oder Speiche eine ulnare oder radiale Ablenkung

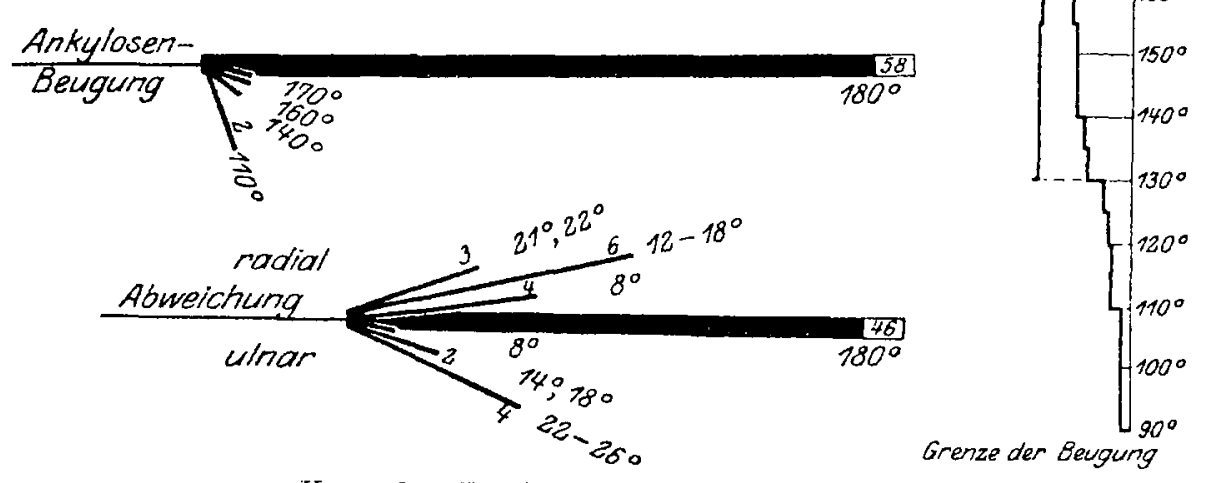

Kurve la. Handgelenk.

Kurve $1 \mathrm{~b}$. Handgelenk.

der Hand, und zwar 13 mal radial (3 Fälle zwischen $21^{\circ}$ und $22^{\circ}, 6$ Fälle zwischen $12^{0}-18^{0}, 4$ Fälle um $\left.8^{\circ}\right)$, ulnar hingegen 7 Fälle (4 zwischen $22-26^{\circ}$, je einer mit $18^{\circ}, 14^{\circ}$ und $8^{\circ}$ ). Dabei komte ich beobachten, daß bei einer Zerstörung der Handwurzelknochen meist eine Ankylose in normaler (gerader, gestreckter) Stellung erfolgte; waren an der Verletzung aber mehr die Unterarmknochen beteiligt, so entstand bei geringer Schädigung eine Ankylose in radialen oder ulnaren Anteil in meist gerader Stellung; bei größerer Zerstörung, wobei oft ein Teil des Knochens fehlte, trat aber die radiale oder ulnare Ablenkung, Subluxation oder Paralellverschiebung ein. Durch die bei der Ankylose eintretende starre Verbindung der Speiche mit der Elle (durch die Kallusverbindung mit der Handwurzel) wird aber als schwerwiegendste Folge für die Funktion die Pro- und Supination aufgehoben!

Unter den $\breve{0} 0$ Fällen die mit einer gewissen Beweglichkeit ausgeheilt sind, konnte ich als Durchschnittsausmaß der Bewegung $33^{\circ}$ feststellen. Dies 
war hauptsächlich eine Beugung aus der geraden Streckstellung (Hand in der Verlängerung der Unterarmachse); ein Heben (Dorsalflexion) ist nur in $1 / 5 \mathrm{~d}\left(\mathrm{I}^{\prime}\right.$ Fälle möglich, während sich eine Reihe von Bengckontrakturen fanden, die überhaupt die Hand nicht bis in die Verlängernng der Unterarmachse heben konnten.

Weder die Ankylosen noch die Kontrakturen allein bedeuten ạn sich einen für die Funktion sehr großen Ausfall, da wir ja auch sonst bei schwereren Arbeiten das Handgelenk willkürlich feststellen; schwer schädigend ist jedoch bei Anky-

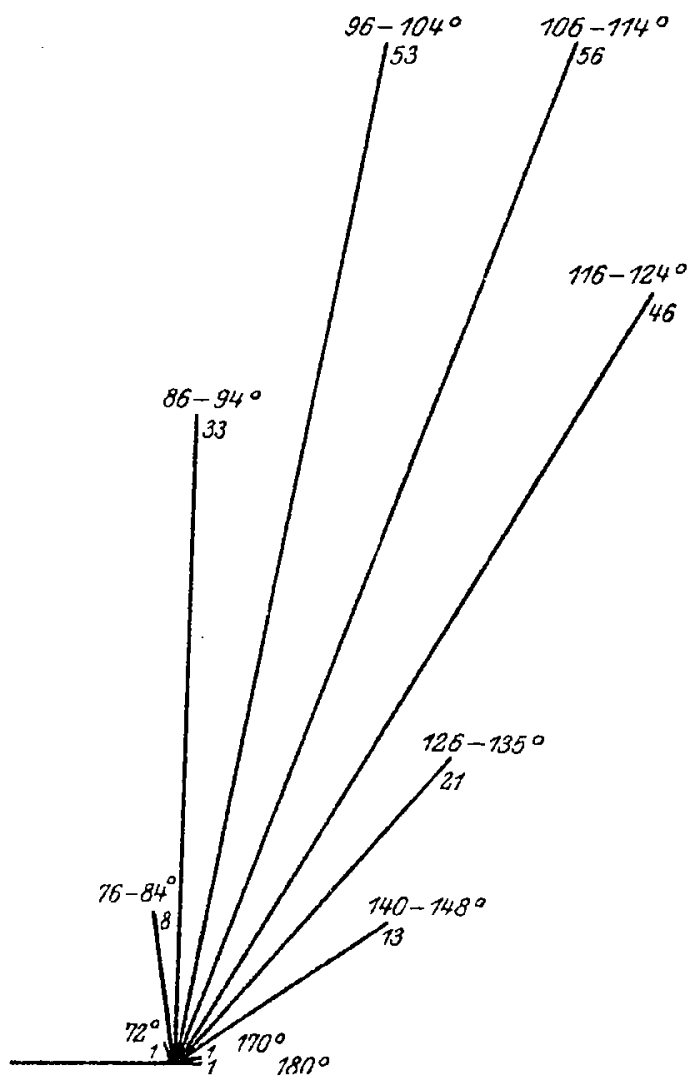

Kurve 2a. Ellbogen. losen die Behinderung der Dreh bewegung, während die Beuyestellungen auch bei Kontrikturen schon an sich weren Veruninderung der Kraft des Faustschlusses für eine ordentliche Funktion ungünstig sind. Nun sind aber die Handgelenksverletzungen meist mit ausgedehnten Zerstörungen und Verwach sungen der Beuger- und Streckersehnen für die Finger, oft auch mit Verletzung des Ulnaris oder Medianus verbunden, durch deren Schädigung das wichtige 'Tastgefühl aufgehoben wird und der hierdurch bedingte Funktionsausfall ist weit wichtiger und größer als die Beeinträchtigung durch die Einschränkung dor Handgelenksbeweglichkeit. an sich.

\section{Ellbogengelenk.}

(Kurve 2a, b.)

Das Ellbogengelenk war in 530 Fïllen direkt betroffen; die Zahl der Füle macht somit, $34,46 \%$ der Gesamtzahl aus. An chirurgischen Eingriffen sind meist nur Inzixionen an allen Stellen des Ober- und Unterarmes zu verzeichnon; rine systematische Drainage nach hinten oder innen komte ich im allgemeinen nicht feststellen. Ebensowenig eine Durchbohrung des Olelkranon; wie dies von Doberaner vorgeschlagen wurde. Nur ist anch mir anfgefallen, daß in jenen Fällen, bei denen durch den Schußdefekt der unbehinderte Sekretalbfluß, nach hinten und innen gesichert war, die Bohandlungsdauer eine wesentlich kürzere war als bei denen mit regellosen Inzisionen und Drainagon meist an der radialen und lateralen Seite behandelten Fillen, die oft nach Jahren noch eiterten. Dagegen scheinen Resektionen im Ellbogengelenk sehr häufig gemacht zu wer- 
den, denn ich konnte 47 Fälle (fast $9 \%$ ) zusammenstellen. In 15 weiteren Fällen war eine schwerste Zertrümmerung der Gelenkenden durch den SchuB orfolgt, wobei hier die Splitter fast ausnahmslos entfernt waren. Von diesen 63 Fälen sind nur 18 mit fester Ankylose ausgeheilt, während bei 44 ein Schlottergelenk resultierte. Ich muß hier ausdrücklich feststellen, daß die meisten dieser Schlottergelenke durch die radikale und geflissentliche Entfernung alles dessen, was Knochen und Periost beißt, bedingt sind, also künstlich herbeigeführt wurden. Ob die Notwendigkeit hierzu gerade beim Ellbogengelenk, das anscheinend gegen Infektionen lange nicht so empfindlich zu sein scheint, wie z. B. das Knie, wirklich immer bestanden hat, hängt natürlich sehr von den besonderen Umstiinden des Einzelfalles ab, wird aber einigermaßen zweifelhaft, wenn man Fïlle beobachtet, die voll von Geschoßsplitter und abgesprengten Knochen-

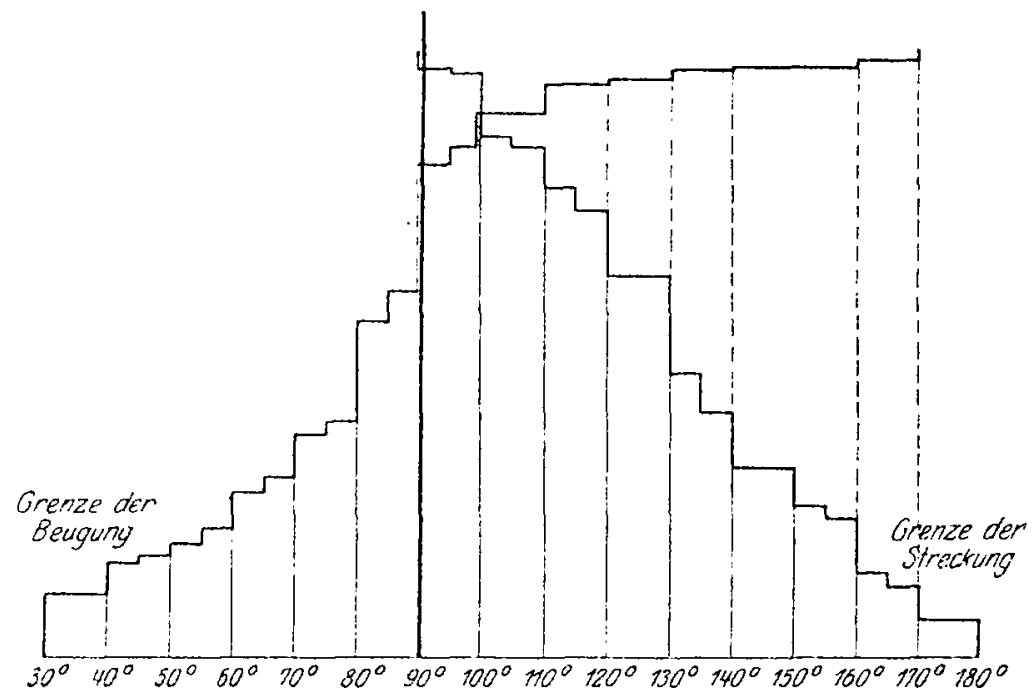

Kurve 2 b. Ellbogen.

teikhen waren mal noch sind, die ebenfalls infiziert in relativ knzer Zeit mit einer festen Ankylose zur Ausheilung kamen (Abb. 1). Ioh konnte vielfach wanz unglaubliche Zortrümmerungen der Gelenkenden feststellen, wobei auch die Diaphysen der beteiligten Röhrenknochen weit hinauf in Mitleidenschaft gezogen waren und die ohne besonderes Zutun des Arztes tadellos und fest ausgeheilt sind und natürlich ein funktionell vicl besseres Resultat ergeben als die Schlottergelenke, die ohne Stiitzapparat ja eine vollkommene Gebrauchsunfühigkeit des Armes im Gefolge haben. Hier gelangt eben die Frage zur Lintscheidung ob funktionell die feste Ankylose oder das Schlottergelenk beim Ellbogengelenk besser brauchbar ist. Ausschlaggebend ist hierfür meiner Ansicht nach einzig und allein der Beruf des Patienten; denn mit einer festen Ankylose kann ich den Arn bis zur Hochhebhalte heben; ich kinn auch schwere Gegenstände heben, tragen, schwingen. Je nach dem Versteifungswinkel kamn bei den Verrichtungen des täglichen Lebens anch noch die gesunde Körperseite des Oberkörpers und der ganze Unterkörper versorgt werden; 
ich komme auf die Zweckmäßigkeit der verschiedenen Versteifungswinkel noch später zurück. Um mit versteiften Ellbogen essen und sich waschen zu können, kurz um ins Gesicht zu gelangen, darf allerdings der Winliel nieht viel über $90^{\circ}$ sein. Aber auch schwerere Arbeiten wie Feilen, Hämmern, Hobeln sind noch möglich; vor allem ist festzuhalten, daß der Patient mit Kraft heben, schieben und ziehen kann. Mit einem schlottergelenk kann der Mamn ohne Apparat überhanpt nichts tun als einen Gagenstand crfassen und ihn haltun. alle Bewegungen aber, die er damit ausführen kann, sind minimal. Dies gilt für alle meine Schlottergelenke, die ich nach Pesektionen gefunden habe; dem ich konnte in der Regel einen Zwischenranm zwischen Ober- und Unterarm

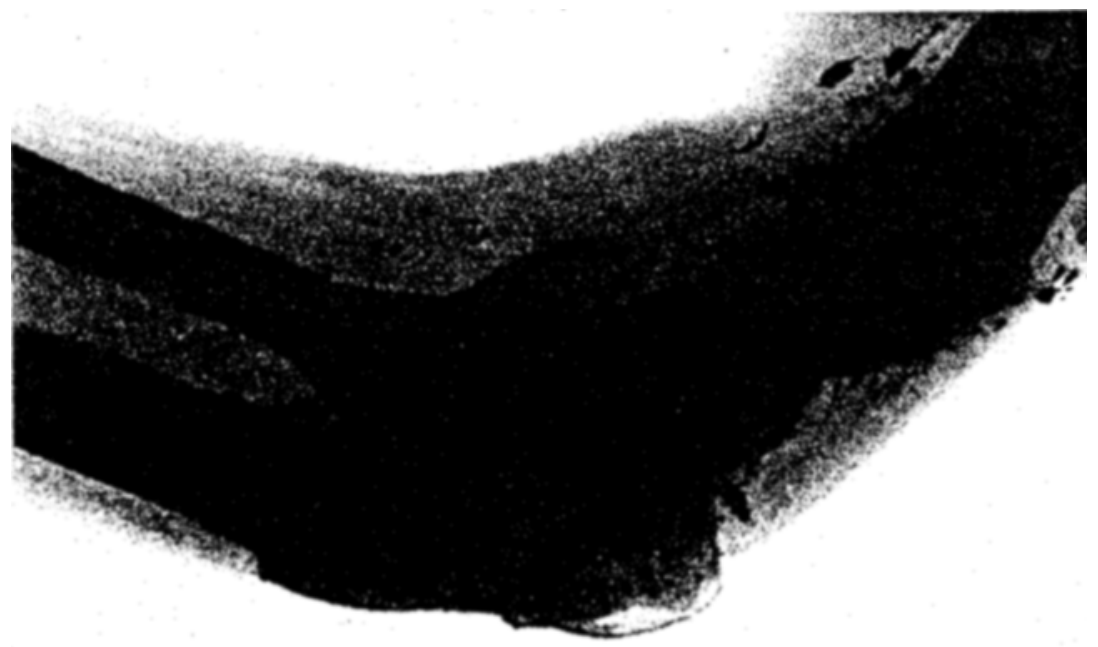

Abb. 3 .

von mehreren Zentimetern oft bis $\%$ 6 $6 \mathrm{~cm}$ und je einmal $61 / 2,8,81 / 2$ und $10 \mathrm{~cm}$ feststellen! 1 )

Mit einem Apparat hat or zwar die Verstellbarlieit in verschiedenen Bengungswinkeln, in einzehen fillen anch aine zewisse alktive Beweglichkeit, so daß er sich bei hiuslichen Arbeiten wird holfen könen, aber es fehlt ihm für die Arbeit die Kraftleistung. Somit dürfte wohl für den manuellen Arbeiter die feste Ankylose anBer jedem Zweifol als diz erstrobenswerto Ziel anzunehmen sein, wïhrend es bei dem Kopfarbeiter immerhin noch zweifelhaft bleibt, ob ihm durch ein schweres sichlottergelenk wirklich mehr gedient. ist als durch eine Ankylose in einem bestimmten Winkcl. Noch ein Umstand spielt dabei eine gewisse Rolle, dic bei den meisten Shohlottergelenken und

1) Um Mißverständuisse zu vermeiden, möhte in hu darat hinweiren, dab zwischen Schlottergelenken nach Resektionen des (iclenkes und ichlotturrelenken nach Frakturen der nächst böheren Röhrenknoshon, wie ich dies \%. B. für den Uberkehenkel und Knie bxschrieben habe, auch funktionell tin grundlegender linterschied ist. (Bruns Batrage zun klinischen (hirurgic 106. Heft 2.) 
schweren Zertrümmerungen oft vorhandene mehr oder minder starke Mitverletzung des Nervus radialis. Da ich aber über eigene Erfahrungen über die Indikationsstellung zur Ellbogengelenksresektion nach direhten Gelenhsverletzungen nicht verfüge, möcbte ich nur erwähnen, daß Hoffmann und Hahn nach eigenen Erfahrungen an einem ziemlich großen Material die Resektion am Ellbogengelenk nicht für notwendig halten und mit entsprechender Drainage sogar funktionell sehr befriedigende Pesultate (Beweglichkeit) erzielen konnten.

In 5 Fällen wurde nur der Padiuskopf reseziert; dadurch entstanden natürlich keine Schlottergelonke, sondern 4 wurden ganz fest, einer heilte mit einer geringen Beweglichkeit aus. Ebenso fand ich einmal eine Resektion nur des medialen Kondyls, die ebenfalls mit $20^{\circ}$ Beweglichkeit ausheilte und schließlich einmal eine Resektion des ganzen Olekranon, wobei nur der Radius mit seinem etwas zertrümmerten Kopf eine feste Ankylose mit dem Humerus einging (Abb. 2). Auffallenderweise stammen die Resektionen des Radiuskopfes aus dem Jahre 1914/15, während die teilweise Resektion des medialen Kondyls,

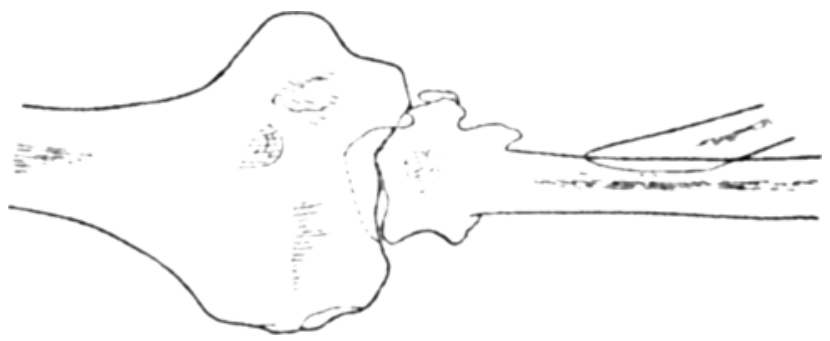

Abb. 2.

die des Olekranon und schließlich einige totale Resektionen, die mit funktionell sehr gutem Resultat (aktive Beuge- und Streckmöglichkeit ohno Apparat) ausgeheilt waren, aus der jüngeren Zeit (1917) stammen.

Außer den 43 Schlottergelenken fand ich 234 Ankylosen; 37 Fille- mit starker Beeinträchtigung der Beweglichkeit; 212 Fälle, bei denen die Beweglichkeit noch wesentlich eingeschränkt war und nux 4 normale Fïlle. Der Versteifungrwinkel bei fester Ankylose, der in den einzelnen Fallen erreicht wurde, bewegt sich im allgemeinen zwischen dem 86. und 126. Grad; und zwar fand ich 33 Fälle zwischen $86^{\circ}-94^{\circ}$ versteift; 53 zwischen $96^{\circ}-104^{\circ} ; 56$ zwischen $106^{\circ}-114^{\circ} ; 46$ zwischen $116^{\circ}-126^{\circ}$. Spitzwinkelige Beugeversteifungen waren ungemein selten, nur 8 Fälle zwischen $76^{\circ}-84^{\circ}$ und ein Fall mit $72^{0}$. Stumpfwinkelige Ankylosen sind häufiger, so zeigten 21 Fälle eine Versteifung zwischen $126^{\circ}-135^{\circ} ; 13$ Fälle zwischen $140^{\circ}-148^{\circ}$ und je ein Fall $170^{\circ}$ und $180^{\circ}$. (Zur Frage der Messungen der Ellbogengelenksversteifungen im Röntgenbilde möchte ich bemerken, daß ich immer den Winkel der Humerusachse zur Ulna genommen habe, weil wir dadurch ron der jeweiligen Pro- oder Supinationsstellung des Radius, wodurch immer ein anderer Winkel entstehen kann, unabhängig sind. Diese Messung ist auch deshalb sachlich richtig, weil ja nur die Una mit dem Humerus die Beugung und Streckung eingeht.) Daraus ist wohl als Regel abzuleiten, daß alle Ellbogengelenksverletzungen in einer ungefähr 
rechtwinkeligen Beugung und leichter Pronation des Vorderarmes grelagrert werden. Als Durchschnittwinkel ergab sich mir 1100. Was den Wert des Versteifungswinkel anlangt, so wäre zu bemerken, daß der rechte Winkel den Vorteil hat, daß der Patient den erfaßten Gegenstand nahe an den Körper heranbringen kann, wenn auch nur hauptsächlich nach der gesunden Seite hin, daß er sich ins Gesicht langen, Grüßen und sich beim Ankleiden wesentlich helfen kann. Auch feinere Arbeiten die näher dem Auge ausgeführt werdeu müssen, sind möglich. Für alle Arbeiten, aber, die weiter weg vom Körper stattiinden müssen, schon zum Zeichnen, aber vor allem bei Betrieben, die eine gewisse Gefahr bedingen, Hantieren an einer Maschine, Arbeiten beim Fouer, an der Esse usw. ist es vorteilhafter, eine leicht stumpfwinkelige Ankylose zwischen $100-120^{\circ}$ zu wählen. Je mohr sich aber wieder der stumpfe Winkel dem gestreckten nähert $\left(150-180^{\circ}\right)$ desto nachteiliger machen sich wieder die Versteifungen bemerkbar, donn jetzt befindet sich jeder erfaßte Gegenstand mindestens $1 / 2 \mathrm{~m}$ von Körper entfernt. Wichtig ist ferner, daß bei allen Ankylosen auch die Pro- und Supination aufgehoben ist (Ausnahmen sind sehr selten), wodureh alle Arbeiten, die eine gewisse Drehbewegung erfordern, wie z. $B$. Schloß aufsperren, Bohren, eine Schraube eindrehen usw. fast unmöglich werden.

Wie die Ankylose zustandekommt, ist meist leicht zu erkennen. Wenn, wie in der Regel, beide Gelenkanteile primär verletzt sind, so geht eben von beiden eine Knochenneubildung aus und bei den nahen Lagebeziehungen zwischen Humerus und Olekranon tritt zwischen beiden fast immer eine feste Verbindung ein. Viel seltener sind jene Fälle, bei denon nur ein Knochen Humerus oder Olekranon betroffen war (der Radius kommt dabei weniger in Betracht) und dann infolge der Knochenläsion eine so starke Kallusbildung aufgetreten war, daß auch der zweite am Gelenk beteiligte Knochen miteinbezogen wurde. Meist ist dabei infolge der Eiterung der Knorpelüberzug auch des gesunden Knochens verschwunden und eine Verknöcherung des Gelenkes eingetreten. Seltener sind auch jene Fälle, wo der Gelenkspalt zwar noch völlig \%u sehen ist, aber ein Kallus des einen Knochens dic Bewegungen auch des anderen fast aufgehoben hat, oder aber, daß zwar die beiden Gelenkenden in ihrer Form vollkommen erhalten sind aber infolge der Fiterung der Knorpel volliommen zerstört und dann sekundär eine knöcherne Verbindung soxusiugen in vollkommenster Adaption der beiden Gelenkenden eingetreten ist. Wie schon erwïhnt, fand ich ein einziges Mal, daß nach starkor Zerstörung der Ulna dor Radius allein eine feste Ankylose mit dem Humorus eingegangen ist. Hiugegen fand ich eine andere bemerkenswerte Tatsache nehrfach, oin sonderbares Widerspiel der Natur. Wenn z. B. der Himnerus knapp oberm Ellbngengelenk stark zerstört worden war und die Gelenkenden infolge der Eiternmg verödeten, und damn vielleicht manchmal zn früh mit den Bowegungen begonnen wurde, so blieb zwar die Ankylose an der Stelle des Gelenkes bestehen, aber an der Frakturstelle des Humerus entwickelt sich eine Pseudarthrose! So fand ich zweimal knapp oberhalb und dreinal knapp nuterhalb des Gclenkes eine Pseudarthrose des Humertus (Abb. 3a, b) bzw. beider Vorderamknochen, die dem Patienten eine relativ sehr gute Beweglichkeit erlaubten, alitives Beugen und Strecken, ̈̈hnlich wie bei einem funktionell günstigen Schlottergelenk, die also 
als eine Art Selbsthilfe der Natur anzusehen ist. Solohe Fäle sind anch rom siandpmat der Pscudarthrosenbehandlung beachtenswert, indem dio Brisciti-

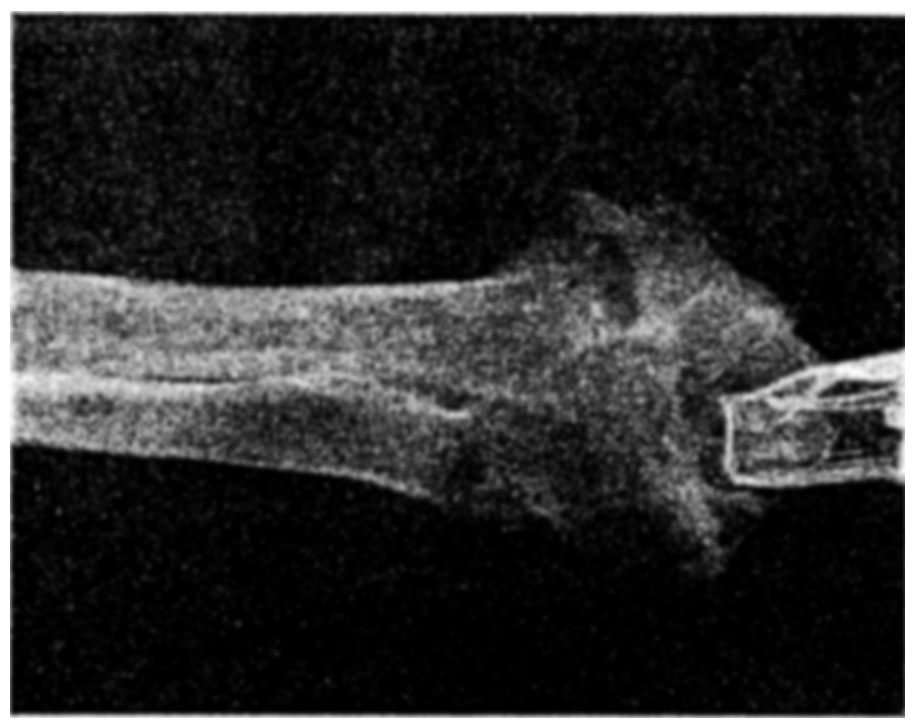

Ahb. $3 \pi$.

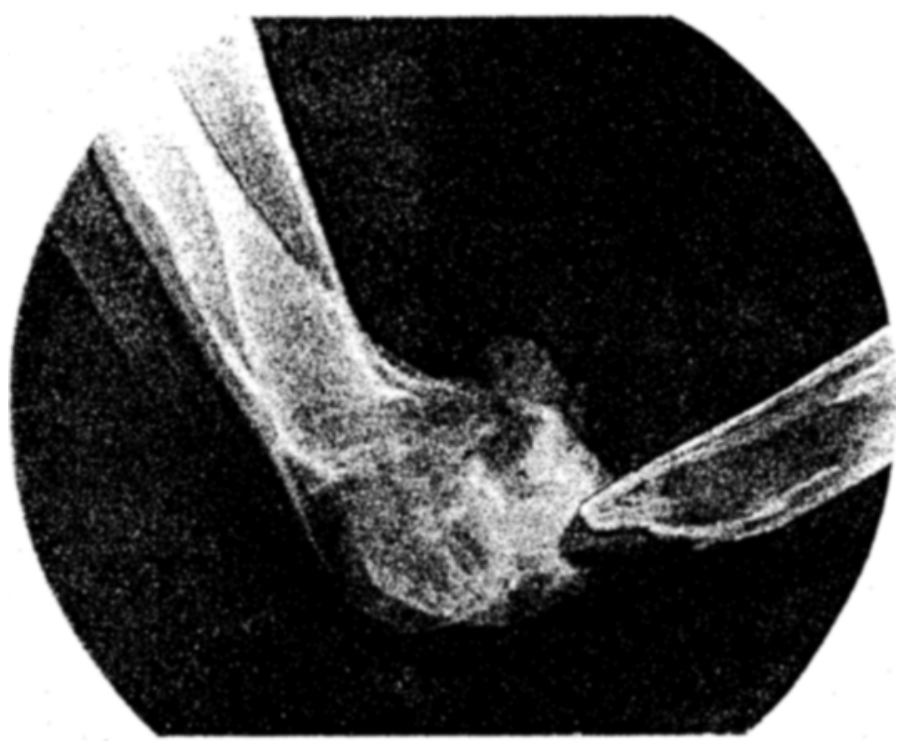

Abb. 3 b.

anug derselben wesentlich schwieriger ist als wenn die Peadapthrose weiter in der 1)iaphyse sitzt und die zugehörigen Gelenke frei sind. Kommen solche operierte Pseudarthrosen irgendwie zu früh an dem starren Verband, so trit dio Psoudathrose mremeidlich wieder anf! Ich wah dies eimmal an einem 
anderwärts operierten Fall und zog für uns die Lehre daraus, daß derartige Fälle eben bis zur vollständigen, absolut festen Verbindung im Verband gehalten werden müssen, wenn die Pseudarthrosenoperation einen Erfolg haben soll. Dies ist mir dann auch in zwei ähnlichen Fällen, bei denen natürlich keine aktiv verwertbare Beweglichkeit, sondern eine schwere Schlotterung an der Pseudarthosenstelle bestand, gut gelungen.

Abgesehen also von diesen 5 Fällen von wirklicher Ankylose mit Bewerlichkeit außerhalb des Gelenkes, waren 253 Fälle $(47,74 \%)$ mit mehr oder weniger Beweglichkeit im Gelenk ausgeheilt. Als Durchschnitt des erzielten Bewegungsumfanges konnte ich $57^{\circ}$ berechnen. Das Maximum betrug außer den 4 normalen Fällen $130^{\circ}$ einmal, das Minimum $5^{\circ}$ fünfmal. Als Ausgangswinkel aus der Beugestellung zur Streckung waren $30^{\circ} 16 \mathrm{mal}, 80^{\circ} 24 \mathrm{mal}$ und $90^{\circ}$ 29 mal festzustellen; die übrigen Fälle verteilen sich fast gleichmäßig auf alle Winkel von $40^{\circ}-130^{\circ}$; nur $120^{\circ}, 140^{\circ}, 160^{\circ}$ und $170^{\circ}$ sind als Ausgangswinkel für die weitere Streckung nur je einmal vertreten. Dagegen sind als Ausgangsstreckwinkel für die Bengung nur die Winkel von $90^{\circ}-180^{\circ}$, und zwar ziemlich gleichmäßig vertreten, nur $130^{\circ}$ und $160^{\circ}$ sind etwas häufiger der Ausgangspunkt für die Beugung, $d$. h. es gibt keine spitzwinkelige Bengkontrakturen, die nicht wenigstens bis $90^{\circ}$ hätten strecken können, aber es gab eine Reihe von Streckkontrakturen, die oft weit entfernt von einer Beugung bis $90^{\circ}$ waren. Als mittlerer Beugungswinkel ist ungefähr $80^{\circ}$, als Mittel der Streckung $130^{\circ}$ anzunehmen. Seitliche Schlotterungen, so daß deshalb Apparate gegeben werden mußten, kamen eigentlich nicht vor.

Im allgemeinen müssen die ausgeheilten Ellbogengelenksverletzungen gleichviel, ob sie num versteift oder beweglich geworden sind oder ein Schlottergelenk darstellen, für die Funktion als immerhin günstig bezeichnet werden, wenn nicht etwa das Schultergelenk gleichzeitig mitbeteiligt war! Alle mit einer gewissen Beweglichkeit aus der Mittelstellung ansgeheilten Fälle geben eine gute Brauchbarkeit. Eine teilweise Gebrauchsfähigkeit geben die Ankylosen, die Schlottergelenke geben eine solche nur mit einem Apparat, wobei die Kraftleistung außerdem sehr gering ist. Am ungünstigsten sind die Streckkontrakturen oder Ankylosen in völliger Streckstellung. Sonst aber wird sich der Patient unter Beihilfe der benachbarten Gelenke immer noch im gewissen Ausmaß helfen und mit dem erhaltenen Arm zufrieden sein können.

\section{Schultergelenk. (Kurve $3 \mathrm{a}, \mathrm{b}$.)}

Das Schultergelenk war 284 mal direkt verletzt, das sind $18,466 \%$. Je nach der Richtung des Durchschusses, der jedoch meist in der sagittalen Ebene erfolgt war, fanden sich die Inzisionen an der Vorder- und Hinterseite; es scheint das Bestreben den Musc. deltoideus zu schonen, immerhin rorhanden zu sein. Seine Intaktheit ist aber sehr oft nicht mehr vorhanden; denn entweder ist der Muskel schon durch die primäre Verletzung stark geschädigt worden, oder aber der ihn versorgende $\mathbb{N}$. axillaris ist durch den Schuß oder die nachfolgende Inzision verletzt worden. Endlich führt ja auch jede langdauernde Uberdehnung des Deltoideus (Fixierung des Armes in Adduktionsstellung) eine kaum mehr z.u beseitigende Inaktivitätsatrophie herbei. Jedenfalls sind Schulterschuß und 
schwerste Deltoideusatrophic fast untrembar miteinander verbunden! Auch die quero Durchtremnung des Deltoideus um das Gelenk ganz breit zugänglich zu machen, komnte mehrfach beohachtet werden; ebenso fanden sich Inzisionen an der Thoraxseite. Im allgemeinen hat man den Ein-

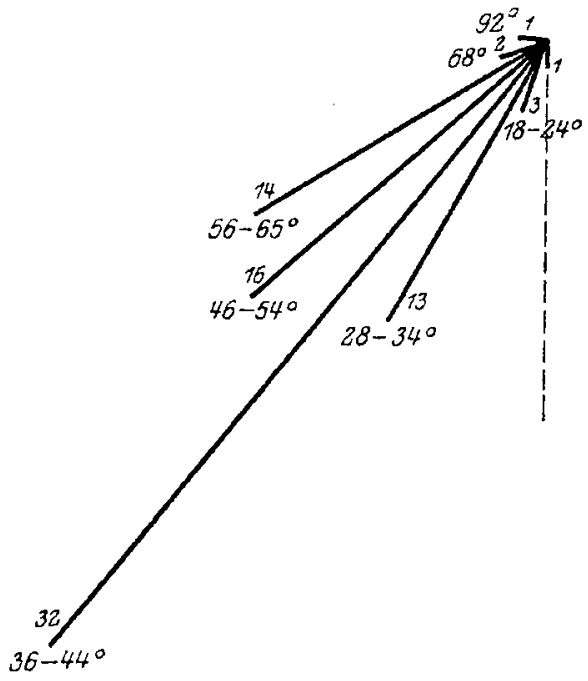

hurve 3a. Schulter.

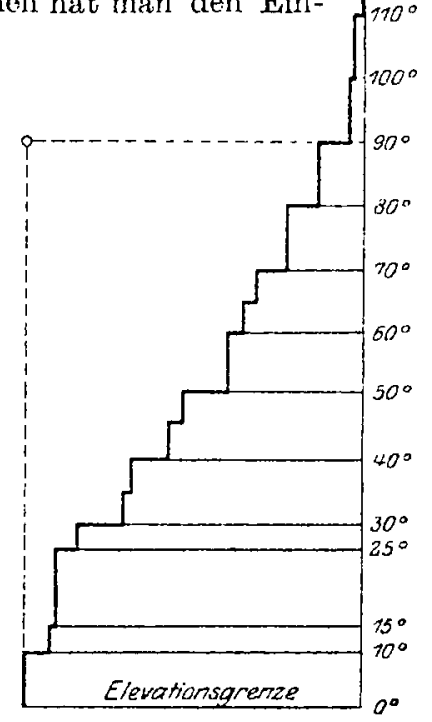

Kurve $3 b$. Schulter.

druck, da $\beta$ die angewand ten chirurgischen Eingriffe ausreichend waren, um der Eiterung Herr zu werden.

Im ganzen waren 20 Resektionen ausgeführt worden, lingegen fand ich noch $22 \mathrm{mal}$ schwerste Zertrümmerungen, so daß daraus allein 38 Schlottergelenke entstanden und nur 4 von diesen Fällen mit einer festen Ankylose ausgeheilt sind. Daher konnte ich $\mathbf{4 6}$ Schlottergelenke der schulter zusammenstellen, die zum weitans größten Teil wieder in der Resektion oder sonstigen radikalen Entfernung hauptsächlich des humeralen Gelenkanteils bedingt sind. Dadurch wurden Diastasen der Gelenkanteile bis 4,5 und $6 \mathrm{~cm}$ je $3 \mathrm{mal}$, je einmal aber sogar 8 , 10 und $18 \mathrm{~cm}$ hervorgerufen (Abb. 4). Die funktionelle Beschränkuvg, die sich aus einem Schlottergelenk der Schulter ergibt, ist so groß und auffallend, daß

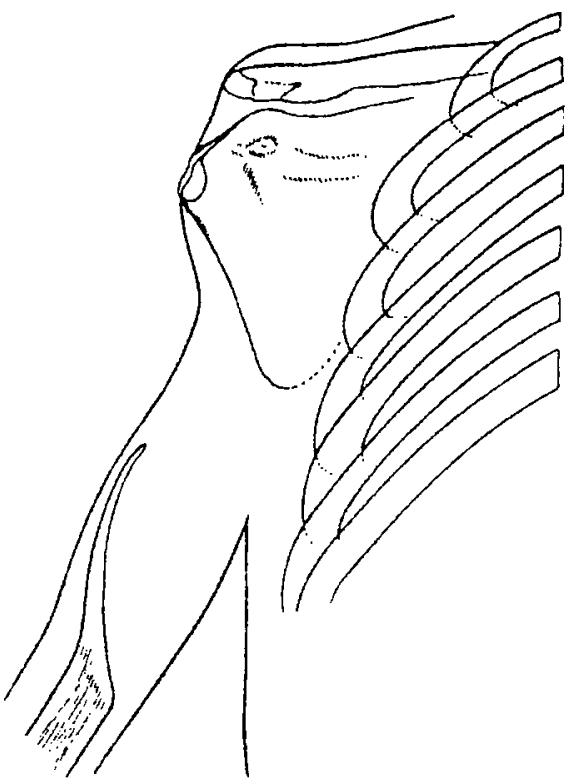

Abb. 4 nicht eindringlich genug darauf hingewiesen werden kann. Der Arm hängt als nicht weiter dirigierbarer Appendix von der Schulter herab, der ergriffene Gegenstand kann trotz intakter Hand und Ellen- 
bogen nur um ein geringes gehoben werden; kein Gegenstand über Brusthöhe kann mehr erfaßt werden und irgendwelche Kraftleistung außer vielleicht Ziehen ist nicht mehr möglich. Ieider kann dieser Funktionsausfall auch durch eiren Apparat nicht wesentlich gebessert werden; lediglich operativ durch eine Arthrodese, durch Einpflanzung des Oberarmes ins Schultergelenk in rechtwinkeliger Abduktion, kann ein Teil des Funktionsausfalles wieder wett gemacht werden. Wir haben diese Operation vielfach mit sehr gutem und befriedigenden Erfolg ausgeführt. Denn gerade bei der Schulter ist die Frage wohl bereits eindentig entschieden, daß eine Ankylose, aber natürlich nur eine solche in ungefähr rechtwinkeliger Abduktion $\left(90-100^{\circ}\right)$ und bei intakter sonstiger Schultergürtelmuskulatur funktionell das erstrebenswerte Ergebnis einer Schultergelenksverletzung darstellt, wenn eben eine aktive Beweglichkeit nicht erzielt werden konnte. Leider sind aber gerade die Ergebnisse meiner Untersuchungen nach dieser Richtung hin nicht befriedigerde.

Die 86 Ankylosen, die ich an der Schulter fand, zeigen meist $36^{\circ}-44^{\circ}$ als Versteifungswinkel (32 mal); eine große Anzahl bewegt sich zwischen 28 und 65 Graden, und zwar 13 Fälle zwischen $28-34^{\circ}, 16$ zwischen 46 und $54^{\circ}$ und 14 zwischen 55 und 65 ; nur ein einziger zeigt $92^{\circ}$ und zwei andere $68^{\circ}$, dagegen 3 nur $18-24^{\circ}$ und einer $0^{\circ}$. Das ist, wie gesagt, ein recht unbefriedigendes Ergebnis, da in allen diesen Fällen nur bei spitzwinkelig gebeugten Filbogen also in unmittelbarer Nähe des Körpers die Hand und damit ein erfaßter Gegenstand bis in die Schulterköhe gebracht werden kann; ein Halten oder Erfassen von Gegenständen, die höher oder weiter vom Körper weg sind, ist also unmöglich. Auch spielt noch der jeweilige Rotationswinkel - ob der Humerus mehr in Innen- oder AuBenrotation im Schultergelenk fest wurde - eine gewisse Rolle, indem bei Innenrotation, wobei also der gebeugte Unterarm $30^{\circ}$ zur Frontalebene dem Körper zugeneigt ist, sich die Hand bei allen Bewegungen in Ellbogengelenk nur ganz nahe am Körper vorbei bewegen kann, während bei Außenrotation, wemn der gebeugte Unterarm in der Sagittalebene schwingt, wicder ein Heranbringen des erfaßten Gegenstandes an den Körper überhaupt unmöglich ist. Als beste Rotationsstellung für Schulterankylosen, die, um nochmals zu betonen, in $90-100^{\circ}$ Abduktion zu erstreben ist, ist eine leichte Außenrotation, so daß der gebeugte Unterarm in Abduktion der Schulter ungefähr horizontal, in Adduktion $30^{\circ}$ von der Sagittalen körperwärts geneigt erscheint.

Bei Messung der Schultergelenksankylose ist natürlich die Stellumg des Schultergürtels, insbesondere des Schulterblattes genau zu berücksichtigen. Ich habe daher in der letzten Zeit nur mehr im Röntgenbild den Abduktionswinkel bestimmt, und zwar habe ich den Winkel gemessen, den der Margo lateral. der scapula mil der Achse des Humerus einschließt. Dabei ergibt sich, daß bei an den Thorax angelegten Oberarm, dieser Winkel bereits $28^{\circ}$ betragen kam; während bei rechtwinkeliger Abduktion dieser Winkel ebenfalls $90^{\circ}$ ausmacht; bei senkrechter Elevation dagegen beträgt er $166^{\circ}$. Da sich bei allen Oberarmbewegungen das Schulterblatt mehr oder weniger mitbewegt, bietet diese Methode der Messung die Möglichkeit der Feststellung der wirklichen Bewegung des Humerus im Schultergelenk.

Während sich nun alle anderen Celenke immer in einem gewissen Gleichgewichtszustand befinden und sich von selbst in ihrer Mittelstellung einstellen, 
hat das Schultergelenk gegen die ständig wirkende Schwere anzukämpfen; daher ist die Puhigstellung des Schultergelenkes nicht seine physiologische Mittelstellung (eine Abduktion von $60^{\circ}$ ), sondern die dem Körper angelegte Adduktionsstellung, die der Funktion so sehr abträglich ist. Wenn also sonst zur Ankylose eine feste Verbindung beider Gelenkanteile notwendig ist, genügt schon eine verhältnismäßig geringe Bewegungsbehinderung der Schulter, um bald die Adduktionsstellung absolut zu fixieren. Wir sehen also die Adduktionsversteifung schon in Frscheinung treten, wenn nur eine Verengerung im Gelenkspalt röntgrenologisch nachzuweisen ist oder es genügt das Auftreten von kleinen verbindenden Bälkchen zwischen Kopf und.Pfanne oder von vorstehenden Knochenzicken am einen oder dem anderen Gelenkanteil. Schließlich aber findet sich die feste knöcherne Verbindung, wenn beide Gelenkanteile gleichmäßig durch die Verletzung betroffen wurden, der Knorpelüberzug zerstört wurde und statt dessen feste knöcherne Kallusverbindung beider Knochen eingetreten ist. In allgemeinen gilt wieder die Beobachtung, daß die Ankylose eintritt, wenn die Gelenkanteile des Schultergürtels verletzt wurden, ebenso wenn nur der Humeruskopf beschädigt wurde, daß aber meist ein Schlottergelenk entsteht, wenn eine stärkere Zerstörung des Humeruskopfes erfolgt ist. Jedenfalls ist festzuhalten, da $B$ im allgemeinen alle Versteifungen nach und nach zu einer Adduktionskontraktur führen wenn nicht mit eiserner Konsequenz die Lagerung des Oberarmes bis zur festen knöchernen Ankylose in einem gewissen Abluktionswinkel erfolgt ist. Ist der Arm in roller Adduktion versteift, so ist er völlig unbeweglich; aber je nachdem die Ankylose in mehr oder minder großer Abduktion erfolgt ist, ist trotz der Ankylose eine gewisse Bewegung des Armes infolge Mitbewegung desselben mit dem Sichultergürtel möglich, und zwar ein Heben und Senken bis ungefähr $90^{\circ}$, da wir den Schultergürtel um ca. $90^{\circ}$ drehen können. Aber nur wenn die Abduktionsánkylose innerhalb dieser $90^{\circ}$ erfolgt ist, können wir auch den Arm wieder bis zur Abduktionsebene heben und durch seine Schwere dem Körper wieder anlegen. Lst die Ankylose in mehr als rechtwinkeliger Abduktion erfolgt, was bei unseren Fallen kaum der Fall war, so kann der Arm meist nicht mehr bis zum völligen Anlegen an den Körper herangebracht werden. Daher müssen wir auch bei unseren Operationen infolge Schlottergelenkes der Schulter, das wir ja am besten durch Arthrodese heilen, darauf achten, den Arm nicht zu stark zu abduzieres, weil er sonst dauernd vom Körper absteht und dadurch den Patienten behindert.

Nur bei ganz gut erhaltener Muskulatur und nicht zu starker Verkürzung und Zertrümmerung des Humerus kann bei einem Schlottergelenk auch ohne Operation eine gewisse aktive Beweglichleit erzielt werden, die ich aber in nennenswertem Ausmaße nie vorgefunden habe.

Abgesehen also von dieser Beweglichkeit, die ohne vorhandenes Gelenk stattfindet, fand ich noch 152 Fälle $(53,53 \%)$, die trotz der Gelenkverletzung eine gewisse Beweglichkeit behalten haben mit einem Durchschnitt von $51^{\circ}$; somit weniger als beim Ellbogen. In 40 Fällen war sie sehr stark eingeschränkt und betrug nicht mehr als auch bei einer Ankylose in Adduktion durch Schultergürtelbewegung erzielt werden kann (gegen $30^{\circ}$ ), in 107 Fallen war sie größer, erreichte aber nur mit 4 Fällen mehr als $90^{\circ}\left(2\right.$ mit $110^{\circ}$ und je einen mit $100^{\circ}$ 
und $\left.120^{\circ}\right)$; im übrigen waren die Fälle auf die einzelnen Winkel riemlich gleichmäßig verteilt. Außer 5 normalen Fällen reichte die Beweglichkeit also nicht entfernt an die Norm heran und genügte nur den bescheidensten Ansprüchen an die Funktion. Ich habe lediglich die aktive Abduktion bzw. Elevation meiner Statistik zugrunde gelegt, weil die Bewegungen in der Frontalebene nach meinen Beobachtungen immer den größten Ausschlag gaben.

Es ergibt sich also für die Gelenkverletzungen der Schulter, daß sie für die Funktion und vor allem für die spätere Arbeit des Patienten meist ziemlich ungünstige Erfolge aufweisen, die auch durch Apparate nicht wesentlich gebessert werden kïnnen und selbst operativ können wir meist durchaus keine idealen Erfolge erreichen.

\section{Sprunggelenk. (Kurve $4 a, b$.)}

Das Sprunggelenk war in 232 Fallen, das sind 15,084\%, direkt betroffen. An chirurgischen Eingriffen, die zur Behebung der Iufektion vorgenommen

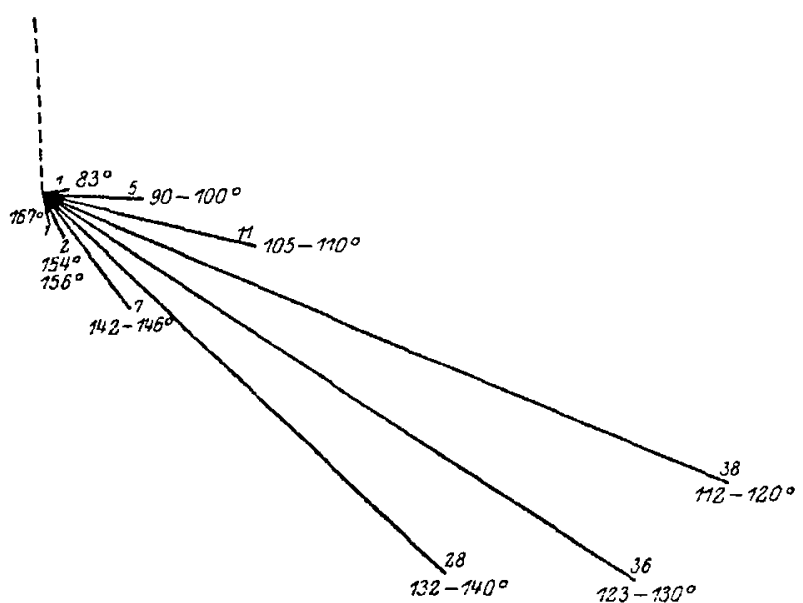

Kurve 4a. iprunggelent.

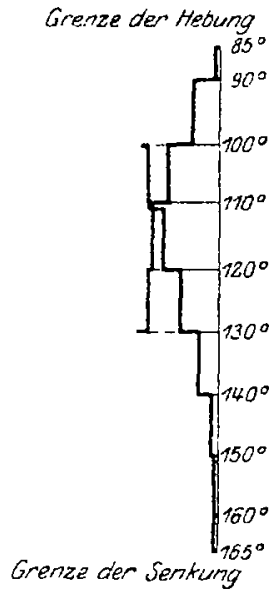

Kiurve 4 b. Sprunggelenk.

worden waren, sind Längsinzissionen über beide Knöchel und übor den Fußrüst die Regel; damit seheint in vielen Fällen das Auskommen grefunden zu werden, doch sind auch die Operationen am knöchernen Gelenlianteil öfter zu beobachten. $12 \mathrm{mal}$ ist das Sprunggelenk reseziert worden, $10 \mathrm{mal}$ wurde der Talus entfernt; davon 8 mit fester Ankylose. Einmal wurde der Kalkaneus reseziert.

Fast alle nennenswerten Verletzungen des Sprunggelenkes führen zur Ankylosenbildung. Dies ist in erster Linie bedingt durch die eigentümliche Form des Gelenkes, indem die Unterschenkelgabel den Taluskörper weit umgreift; somit werden fast immer beide Gelenkanteile gleichzeitig getroffen, wodurch dann eine knöcheme Verwachsung derselben entsteht ( $\mathrm{Abb}$. 5), dann führt aber auch die größte Zerstörung, wenn sie nicht eine Amputation bedingte, immer zur knöchernen Versteifung, wohl auch deshalb, weil hier die Splitterentfernung nicht so fleißig geilbt zu werden scheint, wie bei anderen Gelenken. 
In vielen Fällen dürfte aber wohl auch die Funktion etwas dazu beitragen, indem eben dort, wo eine vollkommene Versteifung durch die Verletzung noch nicht erfolgt war, durch den Reiz der ständigen Belastung beim späteren

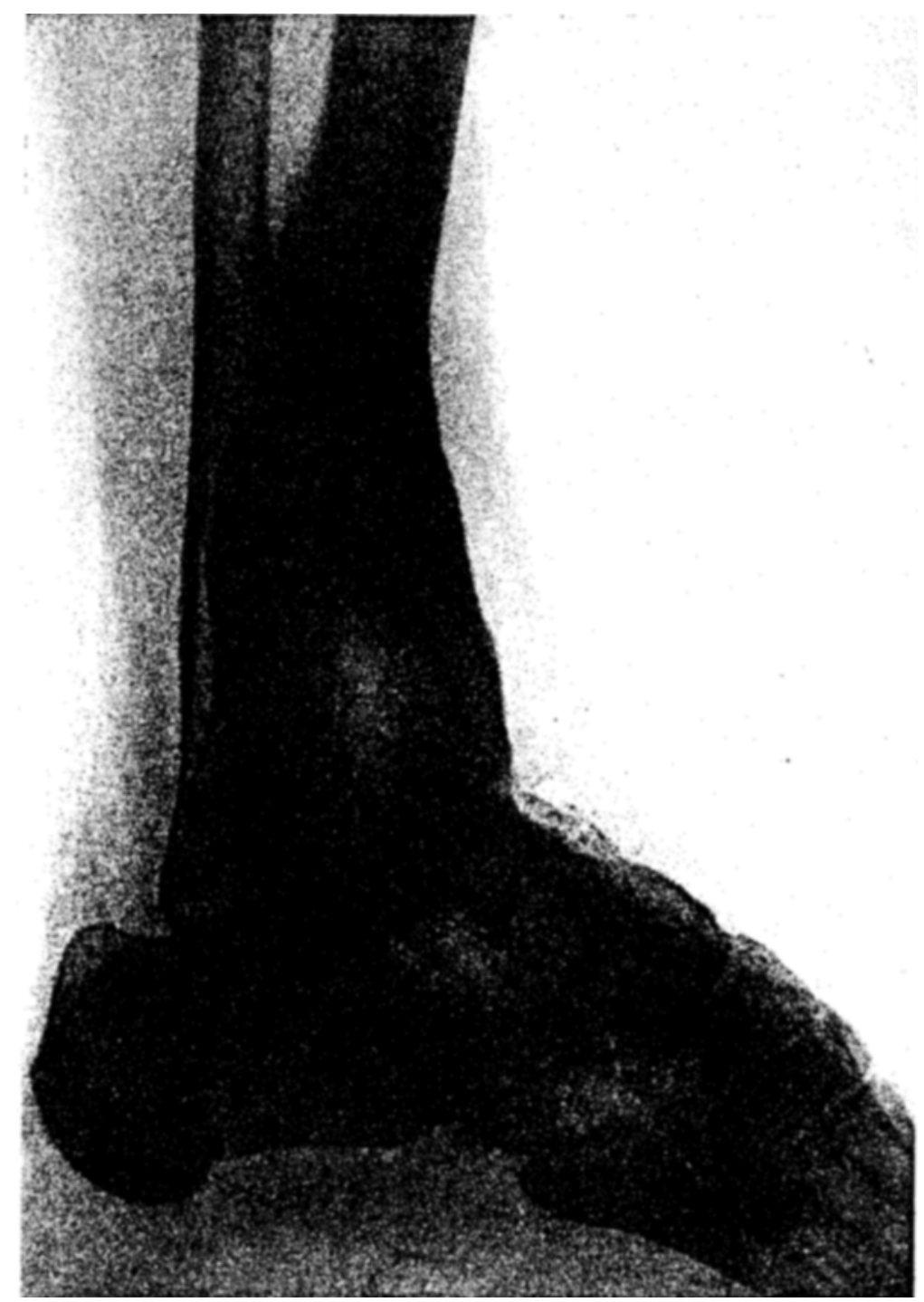

Abb. 5 .

Gehen eine feste Kiallusbildung eintritt. Eine teilweise Versteifung kann eintreten, werm nur clas untere Sprunggelenk verletzt wurde; aber anch hier greift der septische Prozeß meist auf das obere Gelenk über und verödet es. Wir sehen also bei den direkten Verletzungen des Sprunggelenkes den größten Prozentsatz an Versteifungen nämlich $60,78 \%$. Nur ganz ausgedehnte Resek- 
tionen des ganzen Mittelfußes (nach Wladimiroff-Mikulicz) führen zu Schlottergelenker, wenn sie infiziert bleiben ( $3 \mathrm{mal}$ ), ebenso wie die Resektion nur der Malleolengabel ohne Anfrischung des Talus oder sehlieBlich sehr ausgedehnte Zerstörungen, werm nachträglich die Splitter alle genauestens entfernt wurden (s. o.). Alle diese Schlottergelenke des Sprunggelenkes sind meist nur geringgradig, nicht ein führungsloses Herumpendeln wie beim Ellbogen oder Schulter, sondern mehr eine Nichtbelastungsfïhigkeit, eine den Gebrauch be-

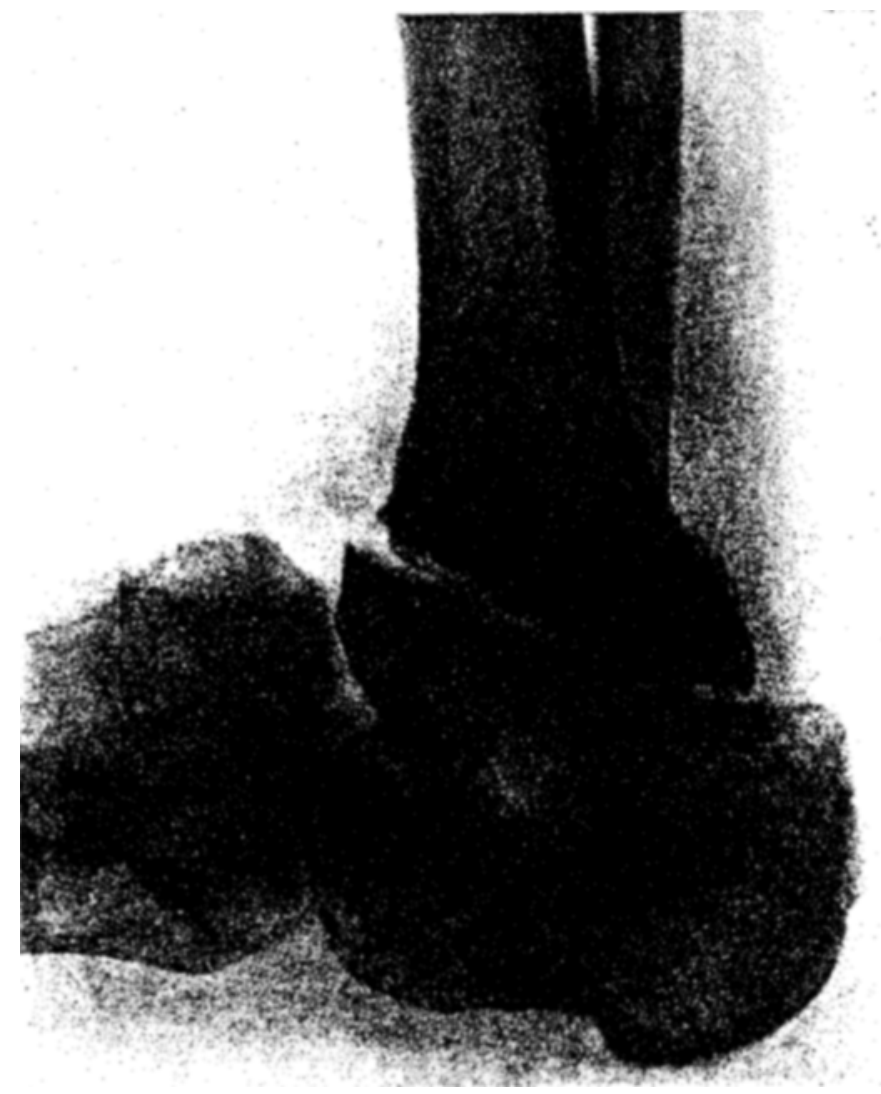

Abb. bia.

hindernde seitliche Nachgiebigkeit. Wir finden also yon 25 Resektionen (Abb. 6, a-c) 17 vollkommene Aukylosen, 4 Fälle mit spurwejer Beweglichkeit, die jedoch für die Funktion ledentungslos ist, und nur 4 Tialle von Sichlottergelenk. Im ganzen fand ich 141 Ankylosen (60.780! ${ }_{i 0}$ ), die alle einen mehr oder minder großen Spitzfuß zeigten. Bei allen Vorletzungen des Sprunggelenkes scheint eben doch auf die spätere Junktion nicht die entsprechende Rücksicht genommen zo werden, denn eine rechtwinkelige Ankylose, wie sio für das $A b-$ wickeln des Fußes vorteilhaft erscheint, fand ich hauptsächlich nur nach Entfermung des Talus und nach einigen Resektionen, im ganzen 17 mal. Die ganze ührige Mehrzahl aber zeigt einen spitzen Winkel ron $112-120^{\circ}$ (38 mal), 


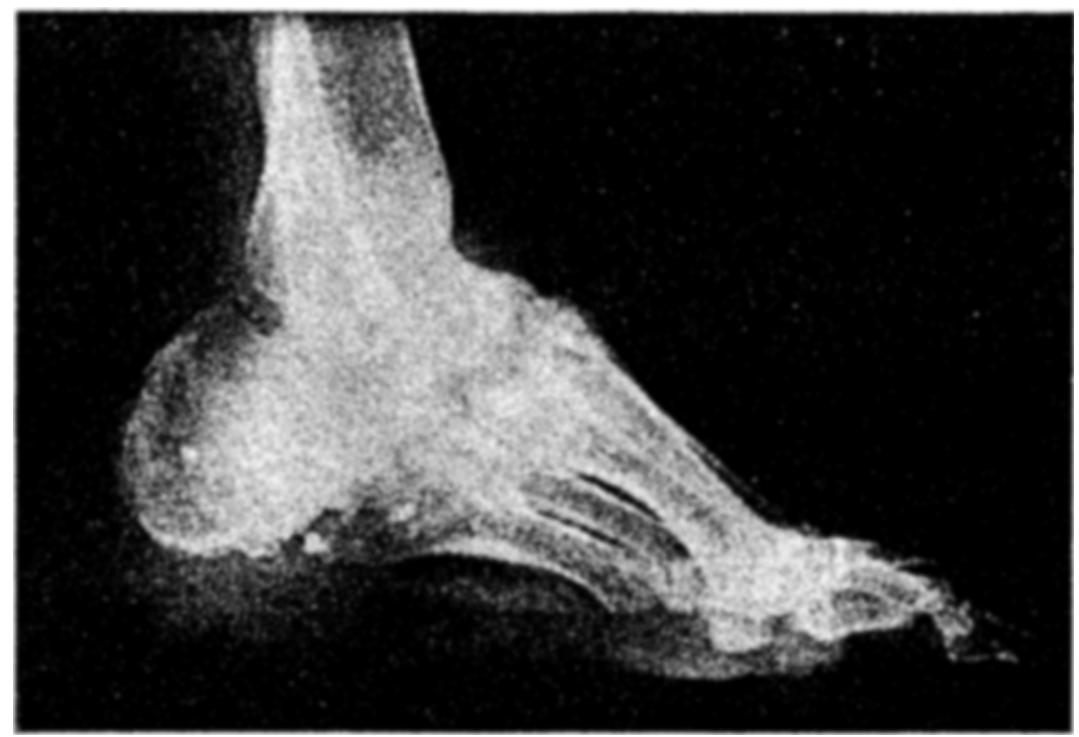

Abh. $6 \mathrm{~b}$

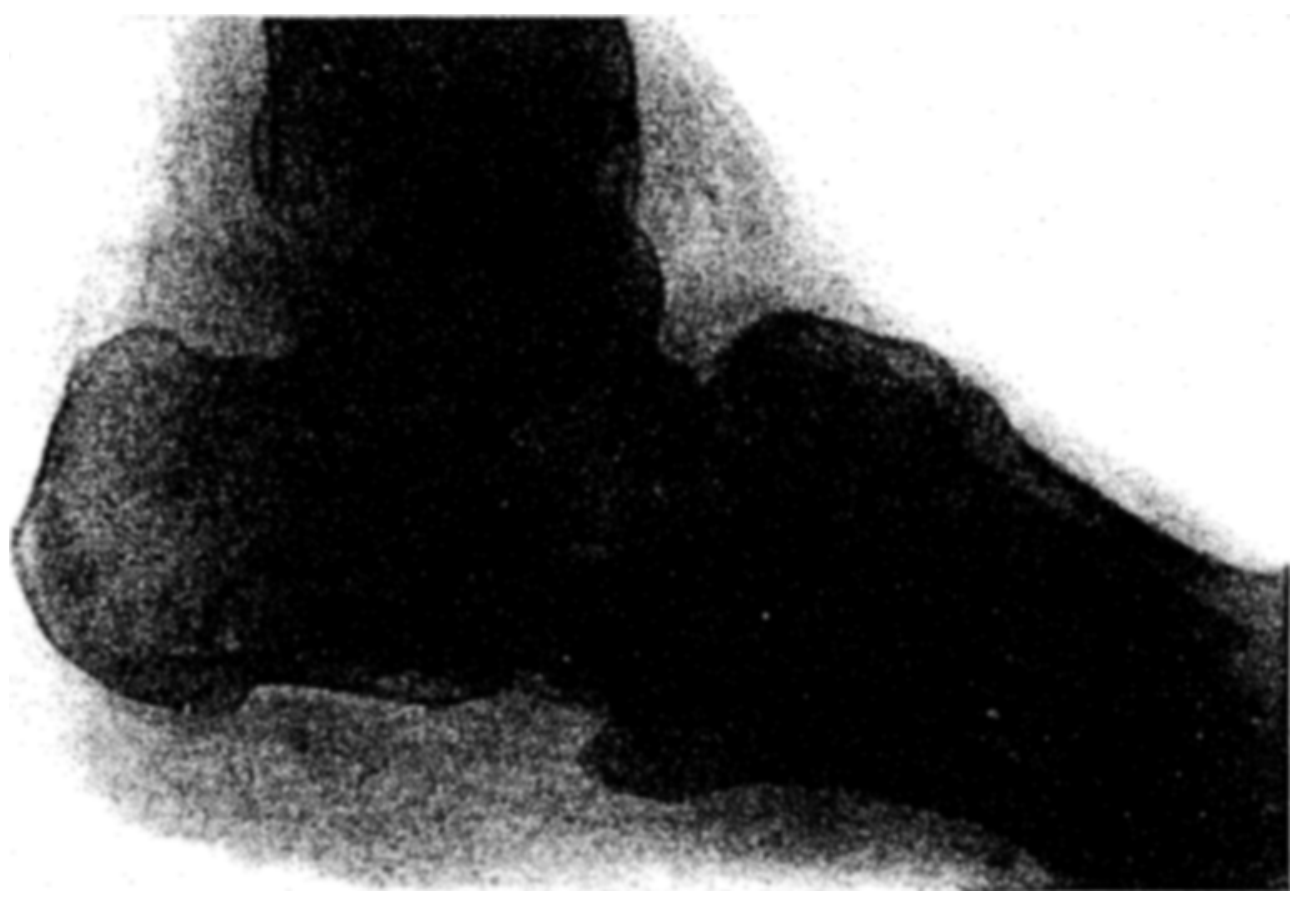

Abb. $6 \mathrm{c}$. 
J23-130 $(36 \mathrm{mal}), 132-140^{\circ}(28 \mathrm{mal})$. Aber auch ganz extreme Spitzfüße von $142-146^{\circ}$ ( 7 Fälle); und je ein Fall von $154^{\circ}, 156^{\circ}, 167^{\circ}$ kamen vor. Fast immer ist außerdem eine leichte Klumpfußkomponente vorhanden, die die Gebrauchsfähigkeit des Fußes nachher noch bedeutend berabsetzt.

Auch für die Bestimmung des Winkels im Sprunggelenk habe ich die Messung im Röntgenbilde herangezogen, und zwar'den Winkel, den die Achse des Unterschenkels (Tibia) mit einer Iinie parallel dem oberen Fußrüst einschließt. Diese Linie ist leicht festzustellen und beträgt der Winkel $115^{0}$, wenn der Fuß rechtwinkelig zum Unterschenkel steht. Von allen meinen Zahlen (bei

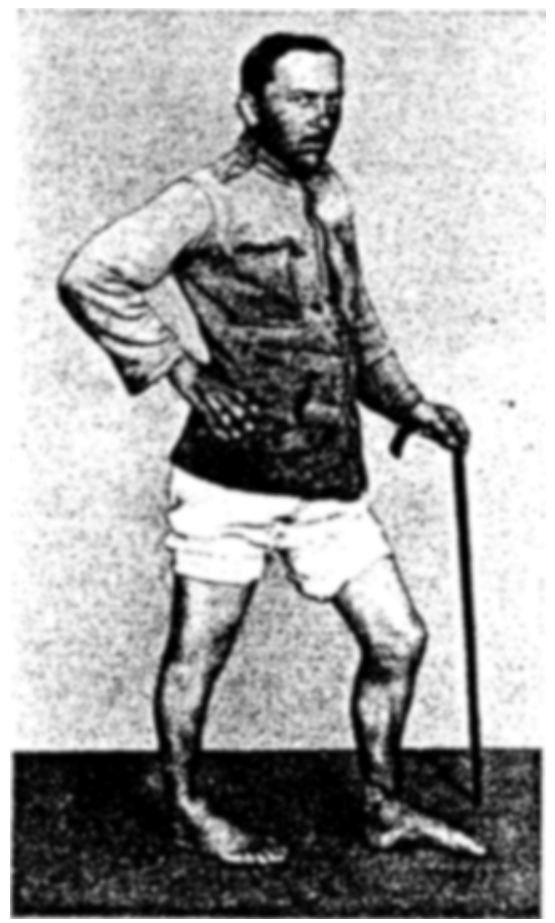

Abb. $7 a$.

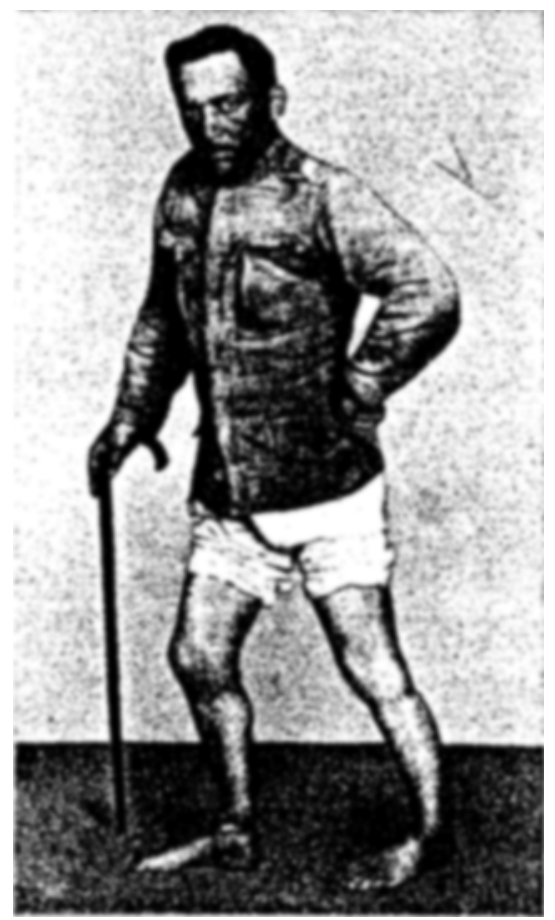

Abb, $7 b$.

Ankylosen) sind somit $25^{\circ}$ abzuziehen, wenn man die gewöhnliche Messung nach der äuBeren Stellung des Fußes damit vergleichen will.

Die kleinere Anzahl von Fällen $(87=37,50 \%)$, die mit einer Durchschnittsbeweglichkeit von $26^{\circ}$ ausgeheilt sind, zeigen aber fast alle eine Spitzfußstellung. Während nur 4 Fälle einen normalen Befund ergaben, war in 56 Fällen die Beweglichkeit nur mäBig eingeschränkt aber für die Funktion ausreichend (Abb. $7 \mathrm{a}, \mathrm{b})$, hingegen in 27 Fiallen geringer als 20 Grade, wovon nur 2 aus der Rechtwinkelstellung heraus dieses geringe $M a B$ von Beweglichkeit zeigten, somit für die Funktion als günstig zu bezeichnen sind, während alle anderen eine mehr oder minder starke Spitzfußstellung aufwiesen. Von den genauer gemessenen Fällen zeigte nur einer eine Hebung bis $85^{\circ} ; 6$ Fälle konnten bis $90^{\circ}$ heben; 5 bis $100^{\circ}$; 3 bis $110^{\circ}$ und je einer bis $120^{\circ}$ bzw. $130^{\circ}$. Das Senken erfolgte 1 mal bis $100^{\circ}$; 
4 mal bis $110^{\circ} ; 3$ mal bis $120^{\circ} ; 5 \mathrm{mal}$ bis $130^{\circ} ; 2$ mal bis $140^{\circ}$ und je 1 mal bis 150 und $160^{\circ}$. Das in vielen dieser Fälle durch eine einfache Achillotenotomie eine Besserung der Fußstellung leicht zu erreichen ist, soll nicht unerwähnt bleiben, aber ebensowenig, daß in gar manchem Falle das Vorhandensein tiefer und ausgedehnter Narben ein schweres Hindernis gegen eine Dauerheilung abgeben dürften. Viel wichtiger wäre auch hier, da $B$ bei allen Verletzungen des Sprunggelenkes strenge darauf gesehen würde, daß möglichst bald eine Ruhigstellung in rechtwinkeliger oder leichter Hackenfußstellung erfolgt; denn sonst wird die Schwere der Decke, die Unmöglichkeit einen schmerzhaften Fuß von selbst mit den Zehen nach aufwärts auf der Ferse dauernd zu balancieren, vielmehr die Einstellung des Fußes in seiner Mittelstellung, die wieder eine leichte Spitzfußstellung ist, endlich der Zug der kräftigen und meist intakten Achillessehne und hemach beim Aufstehen auch die Schwere, immer wieder dafür sorgen, daß der Fuß sich in einer das Gehen schwer behindernden Spitzfußstellung einstellt.

Daraus ergibt sich für die Verletzungen des Sprunggelenkes, daß es für die Funktion viel weniger wichtig ist, ob einige Winkelgrade mehr oder weniger gerettet worden waren, als vielmehr in welchem Winkel als Ausgangsstellung der Fuß danernd zum Unterschenkel steht. Ist dieser nahezu ein rechter, so wird der Patient relativ gut gehen, nähert er sich aber mehr dem spitzen, so kann der Fuß für dio dauernde Belastung erst wieder durch eine Sehnen-oder Knochenoperation brauchbar gemacht werden, denn auch die Anlegung eines Spitzfußschuhes und Apparates hat erst dann einen rechten Zweck, wenn es eben wenigstens passiv gelingt, den $F u ß$ in die rechtwinkelige Stelhing überzuführen. Da wir nun bei jedem "Schritt und Tritt" auf die gute Gebrauchsfähigkeit unserer Füße angewiesen sind, so ergibt sich daraus, wie ungehener wichtig es ist, von vormherin für eine möglichst günstige Fußstellung bei Verletzungen des Sprunggelenkes zu achten. Die je nach dem Sitz der Verletzung mehr an der Innen- oder Außenseite auftretende Neigung zu einer Klumpbzw. Plattfußstellung darf natürlich dabei ebenfalls nicht übersehen werden; denn daß ein traumatischer Pes planus für seinen Besitzer eine Quelle von Beschwerden und Schmerzen werden kann, wußten wir bereits aus den Friedenserfahrungen. Das gleiche gilt in vielleicht etwas geringerem Maße von der Varusstellung, die wieder namentlich, wenn sie von einer Schädigung der äuBeren Fußheber begleitet ist, die Gefahr der ständigen Zunahme in sich birgt. Schließlich sind noch Zirkulationsstörungen an den Zehen infolge ausgedehnter Narben am Sprunggelenk und Verletzung des Nerven durchaus nicht selten.

\section{Das Kniegelenk. (Kurve 5a, b.)}

Die direkten Verletzungen des Kniegelenkes nehmen mit 260 Fällen, das sind $16,96 \%$, die dritte Stelle in meiner Statistik ein. Bei der Empfindlichkeit namentlich des Kniegelenkes gegen jede Infektion ist es also von ziemlicher Wichtigkeit, daß doch eine relativ große Anzahl von Heilungen auch bei sicheren Gelenkverletzungen erwartet werden kann. Dies scheint anch die vielfach geäußerte Ansicht, daß man die Amputationen möglichst einschränken soll (Braun), bzw. daß man mit konservativen Maßnahmen doch viele Erfolge cr- 
zielen kann (Hoffmann, Hedaeus usw.) zu unterstützen. Da andere Berichte aber wieder ganz entgegengesetzt lauten, so dürfen wir wohl auch bei den Kniegelenkrerletzungen annehmen, daß schwor infizierte Fälle nur in den seltensten Fällen durch ganz radikale Eingriffe zu retten sind, daß auch hier die äußeren Begleitumstände besonders die Möglichkeit dauernder Ruhigstellung eine große Rolle spielen, und daß natürlich auch die frühzeitige Amputation von Erfolg aber nur quoad vitam begleitet erscheint (vgl. I. Teil der Arbeit).

Da meine Fälle mehr aus den ersten Kriegsjahren (1914-1917) stammen, fand ich nur in einzelnen Füllen eine Drainage nach rückwärts - außer wenn die SchuBrichtung es mit sich brachte - mit und ohne Resektion von Gelenkteilen; relativ oft war nur die vordere Aufklappung mit dem Textorschen Bogenschnitt erfolgt. Auch Inzisionen zu beiden Seiten der Patella fanden sich öfter. Im ganzen wurden 14 mal Reseltionen vorgenommen und betrafen je nach der primären Verletzung eine oder beide Kondylen oder das ganze Gelenk. Infolge dieser oft weitreichenden Entfernung der knöchernon Gelenkanteile heilten 5 Fälle davon 'mit 'schweren Schlottergelenken' aus; nur einer dürfte durch konservative Maßnahmen noch fest werden und ein sechster zeigte nur geringe Be$2184,196^{\circ}$
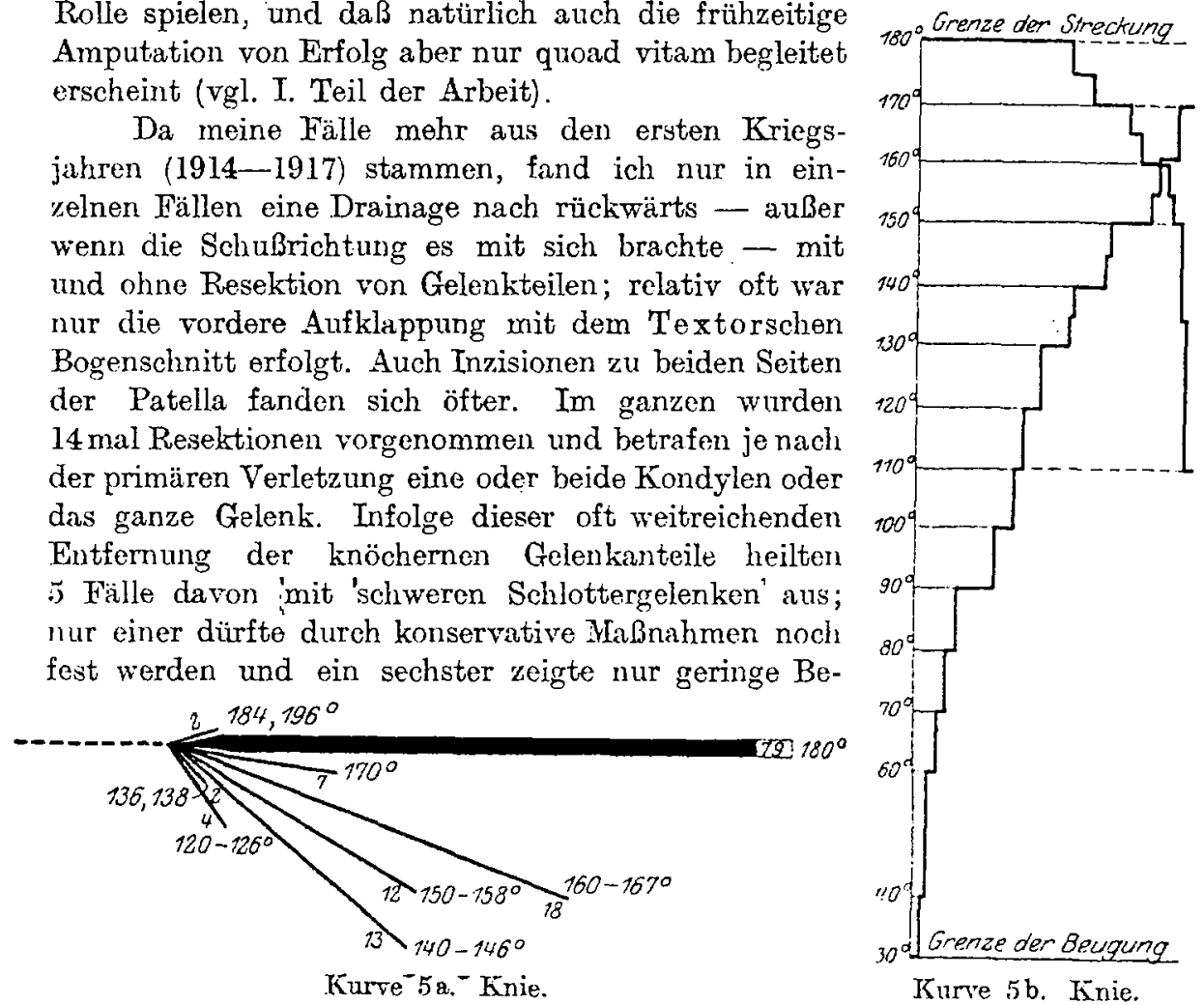

weglichkeit. Hingegen wurde in 8 Fällen eine feste Ankylose erzielt. Zu den 5 Schlottergelenken infolge ron Reselktionen kommen noch 3 infolge starker Zerstörung der Gelenkanteile, obne daß ein nachweisbarer Eingriff am knöchernen Gelenk selbst rorgenommen worden war.

Diese schweren Schlottergelenke, die nur mit Unterstützung eines Schienenhülsenapparates gehfähig werden, sind wohl zu unterscheiden von den viel geringeren Graden von Schlottergelenken nach Oberschenkelbrüchen und zeigten in 3 Fällen eine Distanz der knöchemen Anteile bis zu $5 \mathrm{~cm}$. Sonst war fast immer auch bei schwerster Zertrümmerung der Gelenkkörper eine feste Aukylose meist in funktionell guter Stellung eingetreten; daher beträgt die.Zahl der Ankylosen, die nicht zuletzt auch auf den ständigen Reiz der Funktion zurückzuführen sind, beim Knie 139 also 53,66\% aller Knieverletzungen. Es überwiegen eben beim Bein, das als Stützorgan in ständigem Gebrauch stebt, die festen Ankylosen, in die durchschnittlich $2 / 3$ aller Verletzungen von Beingelenken ausheilen. Oft ist nur der Gelenkspalt bis zur Berührung der Gelenkflächen 
verengt, oder nur die Fminentia intercond. ist knöchern mit dem Femur verbunden, ja in einzehen Fällon ist lediglich in der knöchern mit der Unterlage verwachsenen Patella der Grund für die Ankylose zu finden, oder eine vorspringende Knochenzacks verhindert jede Bewegung. Aber auch einseitign

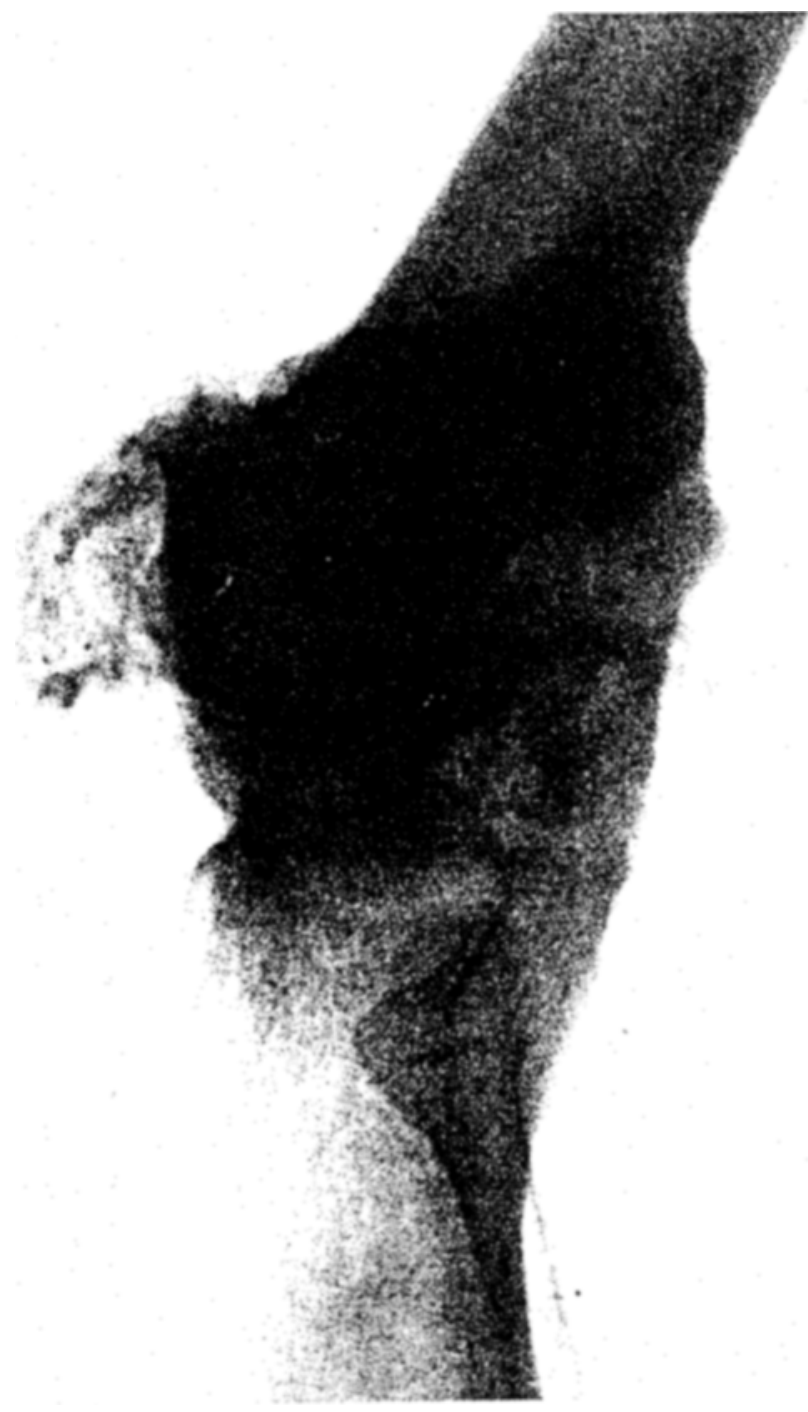

Abb. 8 .

Zerstönung eines Kondyls, der einen Hialfte des Tibia-Winorrens zeirt sich als Grund der knöchernen Vereinigung des Gelenkes bis zu jenen schweren Fählen von vollständiger Zertrümmerung des ganzen Grelenkes, bei denen der Gelenkspalt nur noch auf kure Strecke nachweisbar ist, meist aber ganz fehlt und Fomur und Tibia ineinander äberzuflioßen scheinen. Solche vollständige 
Ankylosen mit Fehlen jeder Gelenkstruktur sind durchaus nicht selten, sie sind oft vergesellschaftet mit Zerstörungen und Knochensprüngen, die weit in die Diaphyse hineinreichen, und weisen nicht selten zahlreiche Metallsplitter zwischen den Fragmenten und in der Umgebung auf. Erfreulicherweise ist die größte Zahl der festen knöchernen Ankylosierungen in Streckstellung erfolgt (79 Fälle), zwei Fälle weisen sogar ein Rekurvatum von $184^{\circ} \mathrm{bzw} .196^{\circ}$ auf; die übrigen sind in mehr oder minder großer Beugung fest geworden. Abor auch hier überwiegen die geringeren Beugungsgrade gegenüber den stärkeren. So zeigen 7 Fälle eine Beugung von $170^{\circ}$, 18 Fälle einen Winkel zwischen 167 bis $160^{\circ}, 12$ Fälle von $158-150^{\circ}$ und nur 13 sind zwischen 146 und $140^{\circ}$ gelegen (Abb. 8). Diese Winkel bedingen schon eine starke Beckensenkung, um

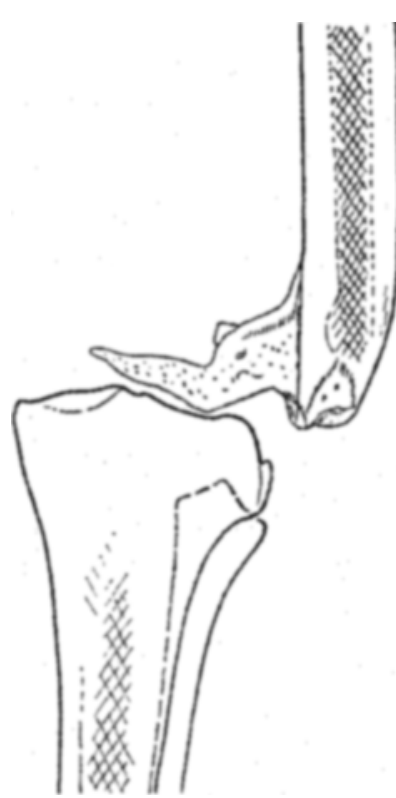

Abb. 9 . mit der Fußspitze noch den Boden zu erreichen und lassen sich durch oine einfache Spitzfußstellung nicht mehr ausgleichen, wie etwa noch die früheren. Je ein Fall aber zeigte nur $136 \mathrm{bzw} .138^{\circ}$ und 4 Fälle lagen zwischen 126 und $120^{\circ}$ ! Fast dreiviertel aller Fialle sind somit in einen brauchbaren gestreckten oder der Streckstellung nahen Winkel ausgeheilt. Leider wird dieses Ergebnis durch das ziemlich häufige Vorkommnis von seitlicheren $\mathrm{Ab}$ weichungen im Sinne einer Varus- oder ValgusStellung sehr stark beeinträchtigt.

Der Grund für die Schlottergelenke liegt weniger in dem Abstand der Fragmente des Femur und der Tibia, wie wir es besonders beim Ellbogengelenk gesehen haben, sondem die ungenügende Adaptierung ist oft der Grund, weshalb die Resektion nicht funktionell befriedigte. So fand ich einmal eine Seitenverschiebung des Unterschenkels gegen den Femur um $7 \mathrm{~cm}$ (Abb. 9), so daß sich die beiden Knochen Femur und Tibia selbst überhaupt nicht mehr berührten. Daß das ein Schlottergelenk schwersten Grades bedeutet, ist klar; aber auch ein Fall von stark schräger Absetzung der Gelenkflächen war für die Belastung ungeeignet und eine andere Resektion, wo die beiden resezierten Knochenflächen $1 \mathrm{~cm}$ voneinander entfernt waren und lediglich die Patella eine knöcherne Verbindung derselben herstellte (Abb. 10), konnte nur mit einem Stützapparat gehen. Auch eine Reihe der später zu beschreibenden seitlichen Verschiebungen bei Ankylosen war eine Folge nicht genügender Adaptierung nach Resektionen.

Die Winkelmessung des Gelenkes im Knie ist sehr leicht, da Femur und Tibia gut durchgetastet werden können und ihre Iängsachse unschwor festzustellen ist. Ebenso verhält es sich mit den Messungen im Röntgenbild. Auch die seitlichen Abweichungen im Sinne einer Varus-oder Valgusdeformität sind unschwer náchzuweisen und zu messen, wenn man z. B. den von mir für die Schlottergelenke im Knie angegebenen Meßapparat ${ }^{1}$ ) der allfälligen Beugung

1) Bruns' Beiträge zur klinischen Chirurgie, Bd. 106, Heft 2. 
gut anpaßt. Die Valgusstellung überwingt mit 16 Fällen sehr stark gegenüber den Varusfälen (7) bei den knöchernen Ankylosen; auch die Grade der Abweichung sind bei Valgus viel stärker. So betrug die größte Abweichung in Sinne eines X-Knie $36^{\circ}$ bei einer gleichzeitigen Beugung von $146^{\circ}$ : der Fall wurde durch eine Osteotomie des Femur wieder gerade gestellt. Ein Fall von $27^{\circ}$ Valgus bei $165^{\circ}$ Beugrung und $21^{\circ}$ Valgus bei gestrecktem Knie wurden ebenfalls durch eine Femurosteotomie in ein gut branchbares Stätzorgan umgewandelt. Dann fand sich noch ein Fall von $16^{\circ}$ Abweichung bei $170^{\circ}$ Bongung, während die übrigen mit je zwei Fillen von $13^{\circ}, 12^{\circ}$ und $10^{n}$ haluptsächlich bei streckankylosen vorkamen. Schlieblich fanden sich noch 3 Fälle ron $8^{\circ}$ Abweichung und je einer von $7^{0}, 6^{\circ}$ und $4^{\circ}$. Dio Varusdeformierung betrug je coinmal $24^{\circ}$ und $I^{n}$ bei Streekstellung, who dak eine operation umbedingt notwendig geworden wäre. Je zwei lialle von $8^{\circ}$ und $5^{\circ}$, winer bei 150" Bongung, ein anderer mit $1966^{\circ} C^{\circ}$ berstreckum, ferner ein Fall von :t" Varus und geleichreitiger Bongung von $165^{0}$ waren ebenfills zum Teil ohne Stritzapparat gehfaihig. Die trumatischen O-Beine sind also nicht nur viel seltener, sondern anch fulktionell wesentlich gimstiger als die X-Boinc. Was die Lirsache für das Entstchen der Varus- oder Talgusstellung anlangt, so war durchaus nicht immer nur eine einseitige Zerstörung des äußeren oder inneren Kondyls nachzuweisen, wie auch die cinscitige Kerstö-

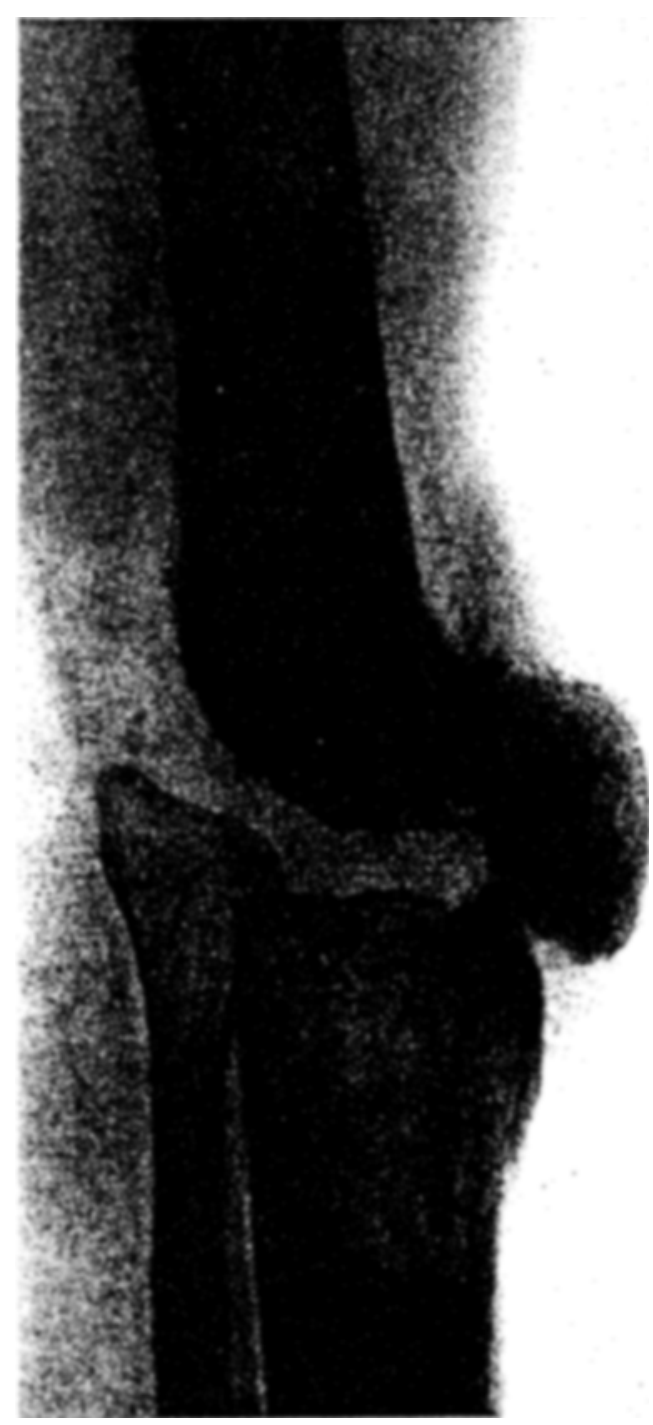

Abb. 10. rung derselben meist keine Abwoichung des Toterschenkels nach der einten oder anderen Seite zur Folge hat. Vielntehr fand ich diese schweren $X$ - und O-Stellungen immer bei starker Zertrümmerung des ganzen Gelenkes, wo eben dann die unrichtige oder ungenügende oder sielleicht ummögliche Fixierungr des Beines zu dieser fehlerhaften stellung geführt haben nuß. 
Eine seitliche Verschiebung des Unterschenkels meist nach hinten oder außen oder beides gemeinsam konnte ich 11 mal nachweisen. Die Subluxation 1)ach hinten (5 Fälle) war meist mit starker Beugung $124^{\circ}$ und $142^{\circ}$ verbunden; die Verschiebung betrug $2-3 \mathrm{~cm}$, die seitliche in 6 Fällen war meist beträchtlicher, zeigte je einmal nach außen $5 \mathrm{~cm}$, nach innen $4 \mathrm{~cm}$ und fand sich meist nach Resektionen.

Die freie Bewegung, soweit sie vorhanden, ist nicht immer geräuschlos; arthritische Voränderungen sind $\mathrm{ja}$ in fast allen diesen Fällen vorhanden und so sind Knirschen und Reiben bei Bewegungen ein sehr häufiger Nebenbefund nach Kniegelenkverletzungen. Auch das Ausmaß der Bewegung ist während der Funktion oft Änderungen unterworfen; so nimmt es bei Uberanstrengungen und Reizungserscheinungen ab, wobei manchmal gleichzeitig Schmerzen auftreten, die in einem Fall so groß wurden, daß der Patient lieber eine starre Hülse trug und auf die nicht unbedeutende Beweglichkeit verzichtete, um nur ja sicher von den Schmerzen befreit zu werden. Im allgemeinen aber ist zu beobachten, daß auch die kleinste Beweglichkeit für den Gang ausgenützt wird. In solchen Fïllen, die leicht zu Reizungen neigen, wird es wohl Jahrc brauchen bis sich für den Gebrauch gleichmäBig gute Verhältnisse eiristellen. Daß wir aber gerade beim Kniegelenk nach jahrelangem Gebralich noch arthritische Veränderungen werden benbachten können, halte ich für gewiß. Trotzdem müssen wir jede Beweglichkeit, die wir uach einer direkten Kniegelenkverletzung feststelleu können, als einen funktionell sehr wertvollen Erfolg buchen, da wir ja wissen, daß die anfangs das Gehen oft sehr erschwerende Quadrizepsatrophie bei ständigem Gebrauch doch, wenn auch langsam, schwindet, wie auch narbige Verwachsungen, soweit sie nicht jede Beweglich keit unmöglich machen, einer langsamen Dehnung zugänglich sein dürften.

113 Fälle, also nur $43 \% \%$ aller Kniefälle sind mit aktiver Beweglichkeit ausgeheilt. Unter diesen befinden sich 77 Fälle, das sind $2 / 3$ mit einem Bewegungsumfang von mehr als $30^{\circ}$ einschließlich dreier Fälle mit normalem Ausmaß gegenüber 36 Fällen mit weniger als 30 bis zu $5^{0}$ freier Bewegung.

Im besonderen betrug das Durchschnittbewegungsausmaß $55^{0}$ und weitaus der größte Teil erreichte die volle Streckung, so 37 von den 72 genau gemessenen. Je 5 von diesen reichten bis $175 \mathrm{bzw}$. 170. Daß die größte Streckung weniger als $160^{\circ}$ betrug, kam nur ganz vereinzelt vor $(6 \mathrm{mal})$. An Bewegungsausmal3 über 90 Grade wurden jo einmal $145^{\circ}, 130^{\circ}$ und $120^{\circ}$ erreicht. 2 Fïlle kamen a uf $110^{\circ}$, nur I Fall auf $105^{\circ}$, dagegen auf $100^{\circ}+$ Fälle und 6 Fäile auf $90^{\circ}$. Von den kleinsten Zahlen sei erwähnt, daß anch viermal $10^{\circ}$ und zweimal nur $5^{0}$ gefunden wurden.

Wenn wir also alle Kniegelenkverletzungen nehmen, müssen wir auch hier die Ergebnisse als nicht ungüustig bezeichmen; denn sie führten entweder zur Ankylose meist in Streckstellung, wobei allerdings auch Deformitäten vorkommen können, die eine spätere Korrektur erforderten oder eines Stützapparates bedurften; oder aber es wurde eine für den Gang meist ausreichende Beweglichkeit erzielt, die freilich für den sitzenden Beruf wohl oft noch störend empfunden werden dürfte. Außerdem besteht die Gefahr, daß spätere Veränderungen die Gebrauchsfähigkeit des Beines einschränken können. 


\section{Hüftgelenk.}

Dic Fälle von clirekter Hüftgelenkverletzung sind nur in der geringen Zahl von 110 Jällen bei uns zur Beobachtung gekommen, betragen somit nur $\pi, 1_{10}^{n}$ aller Fälle. Und da ich die wenigsten von Ihnen selbst untersuchen konnte, sind auch die Endergebnisse nicht mit der gleichen Genanigkeit fundiert wie dio übrigen, daher will ich mich ganz kurz fassen. Auch die Messungen im Röntgenbilde sind sehr schwierig; besonders wenn cine Übersichtwanfnahme fehlte, multen alle möglichen Hilfslinien herangezogen werden, $u m$ wenigstens den Abduktionswinkel bestimmen zu können, da die Bestimnung der Bengung bei nur vorhandener antero-josit. Aufnahme überhaupt unmöglich war. Für den Seliretabfluls war meist die Schubrichtung maßscebend, jedoch wurden Hilfsschnitte in der Glutäalgegend wie an der Hinterseite des Oberschenkels beobathtet. Hatte man sich das Gelenk zugänglich machen wollen, so scheint der seitliche Schnitt äber den Trochanter meist angewendet. Dem eigenartigen Bau des Gclenkes entsprechend, wolurch fast immer beide Gelonkanteile gleichzeitig verletzt worden, ïberwiegen die Ankylosen mit 65 Fällen, das sind $50 \%$. Neben 19 Fällen sehwerster Zertrümmerung des Gelenkrs, wobei meist der femorale Anteil desselben röllig gerstört wurcle, hatte moh !) mal eine Rosektion ausgeführt werden müssen, worats allein 6 Schlottergelenke resultierten, wïhrend 3 mal cine feste dukylose entstand. Dio schwersten Zertrümmorungen hatten $\$$ mal zu cinem Sehlottergelenk groühtt, während $1 \mathrm{lmal}$ eine feste Ankylose eintrat. Wir hatten somit 14 Schlottergelenle, deren Funktionsalssfall aber oft recht gering wal'. Win tiall, wo der ganze ichenkelkopef fehlte, konnte ohne jeden Aprarat tadellos mit katum merkbarem Hinken gehen, marschieren, reiten; bei einem anderen Fall. wo Kopf und Hats rollständig fehiten, konnte ieh den l'rochanter so günstig unter clem oberen Pfannenrand bringen, dan alktiv alle Bewegungen vollkommen frei waren (Abb. $11 a, b, c)$ and von einem leichten Hinken abgeschen der Patient tadellos und stundenlang gehen komte onne jeden Behelf.

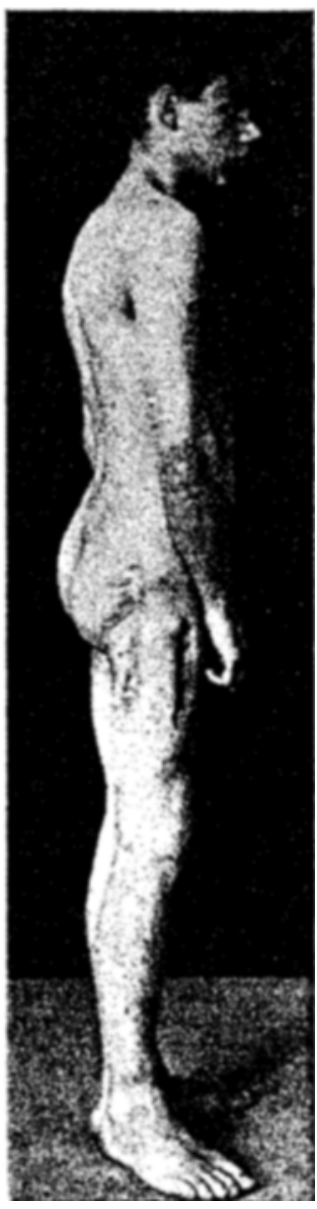

Abls. II i. Tm allgemeinen haben schwerere Verktzungen des Bockens bewegungseinschüinkungen bis zur Ankylose zur Folge, während die Zertrümmerung des Fenuranteiles, namentlich wem infolgo der Zertrümmerung direkt Teile des Kolfes und Halses entfernt wurden, auch öfter zu abnormer Beweglichkeit gefülut haben. Jeder größere Kallus aber muß, wo immer er entsteht, zu starker Bewegmoseinschränkming führen. Daher ist also die Ankylose in der Hüfte: fast ebenso häufig wie im Sjurungrelenk, nämlich fast $2 / 3$ aller Fälle. Der Versteifungswinkel war nur in wenigen Fïllen eine volle Strckstellung; leichte Beugung und Adduktion ist in den meisten Fïllen vorhanden. Einige wenige 


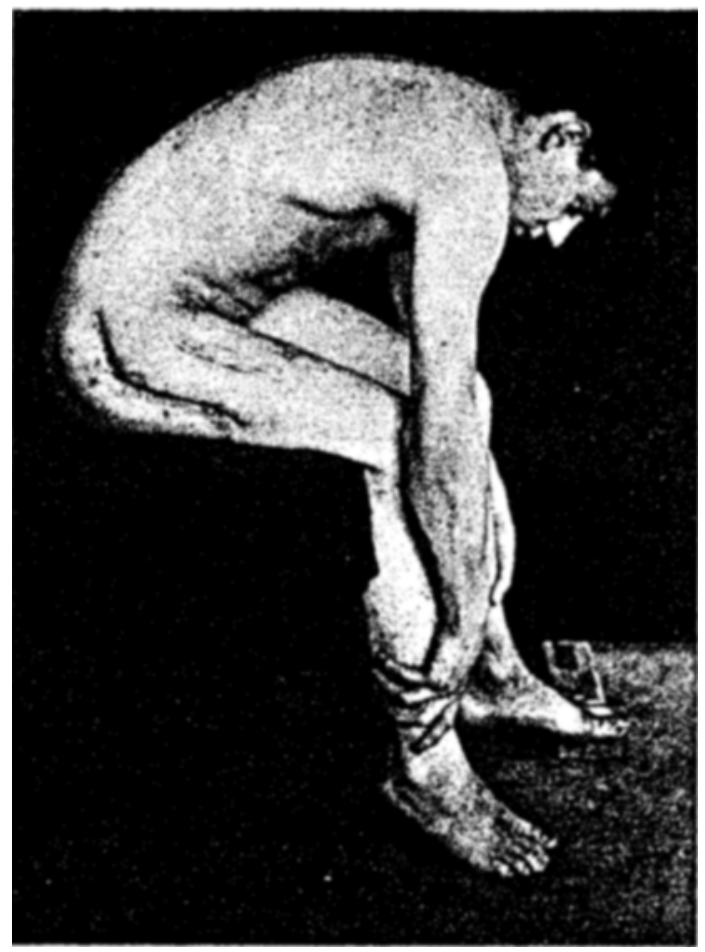

(bb) 11 b.

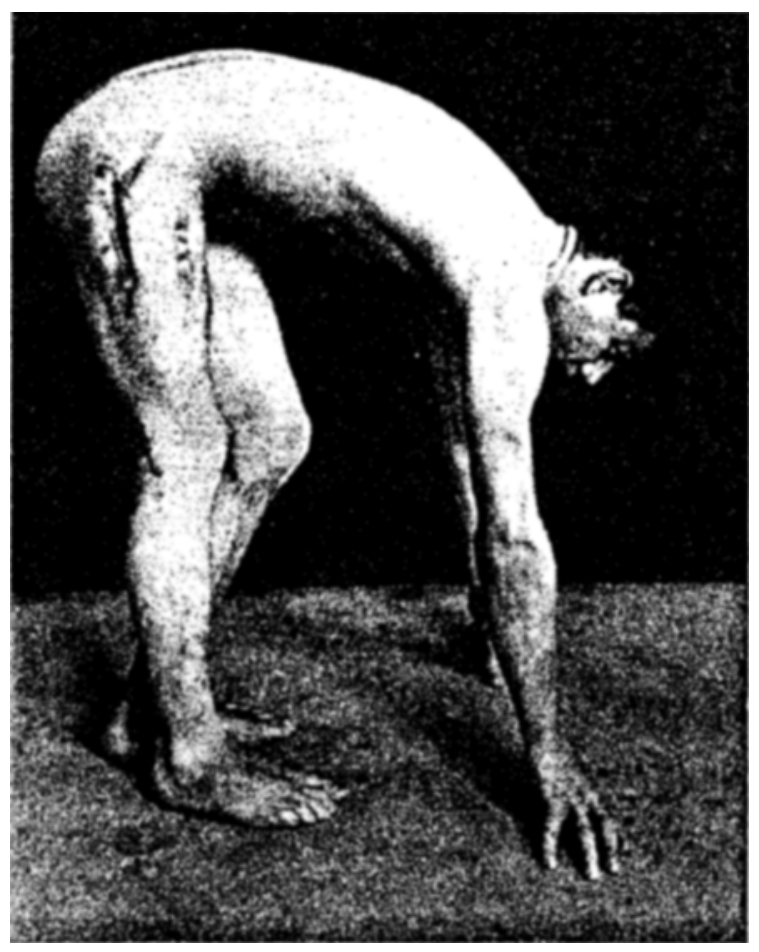

Abb. $11 \mathrm{c}$. 
Fälle (7) zeigten eine Abduktion, und zwar $2 \mathrm{mal} 8^{\circ}$ und 1 mal $24^{\circ}, 12^{\circ}$, $14^{\circ}, 34^{\circ}, 38^{\circ}$. Fälle mit so starker Abcluktion, wie die beiden letzten, lassen sich natürlich nicht mehr durch eine einfache Beckensenkung, die eine nach der kranken Seite konvexe lumbale Skoliose und damit auch eine entsprechende Verlängerung des Beines bedingt, ausgleichen; sie sind daher für die Funktion ebenso unbequem wie die Fälle starker Adduktion, die bei uns $17 \mathrm{mal}$ $5-10^{\circ}$, in 9 Fällen $12-20^{\circ}$ und in 7 Fällen $23-26^{\circ}$ erreichten. Wird nun das Bein auf den Boden gestellt, so hat die dadurch notwendige Schiefstellung des Beckens neben einer Lumbalskoliose, die nach der gesunden Seite konvex jst, noch eine scheinbare Verlängerung des meist ohnehin längeren gesunden Beines zur Folge. Fast immer ist auch eine mehr oder minder große Beugung dabei, die nur durch eine Beckenneigung ausgeglichen werden kann, die wieder eine Lordosierung der Lendenwirbelsiiule bedingt, aber gleichzeitig auch ein ziemlich auffallendes Hinken mit sich bringt. Die Heilung einer Hüftgelenksverletzung in fester Ankylose ist also für die Funktion nicht als sehr günstig zu bezeichnen, abgesehen davon, daß das Sitzen auf normale Art immer stark erschwert ist. Eine ebenfalls noch oft vorhandene Außen-oder Innenrotation des Beines ist von gerungerer Bedeutung, da ja Hüftgelenkverletzungen sehr oft anch mit Lähmungen des Ischiadikus vergesellschaftet sind, die ja noch weit unangenehmere Folgen mit sich bringen.

In 31 Fïllen wurde eine mehr oder minder große Beweglichkeit festgestellt, die wohl oft zur völligen Streckung führte, aber in 12 Füllen wesentlich eingeschränkt war; in 17 Fällen bestand eine für die Funktion nicht mehr stark ins Gewicht fallende Einschränkung und 2 Fälle konnten mit normaler Beweglichkeit festgestellt werden. Auch hier sind die Nebenverletzungen, Lähmungen, Zirkulationsstörungen mit ihren Folgen für den Gebrauch des Beines mit von ausschlaggebender Bedeutung. Jedoch läßt, wie schon eingangs erwähnt, die geringe Zahl der von mir selbst genauer untersuchten Fälle keine allzuweitgehenden Rückschlüsse zu. 